\title{
Hill's spectral curves and the invariant measure of the periodic KdV equation
}

\author{
Gordon Blower ${ }^{a}$, Caroline Brett ${ }^{a}$ and Ian Doust ${ }^{b}$
}

${ }^{a}$ Department of Mathematics and Statistics, Lancaster University, Lancaster, LA1 4YF England, UK

${ }^{b}$ School of Mathematics, University of New South Wales, Sydney, Australia

14th March 2016

Abstract This paper analyses the periodic spectrum of Schrödinger's equation $-f^{\prime \prime}+q f=\lambda f$ when the potential is real, periodic, random and subject to the invariant measure $\nu_{N}^{\beta}$ of the periodic KdV equation. This $\nu_{N}^{\beta}$ is the modified canonical ensemble, as given by Bourgain (Comm. Math. Phys. 166 (1994), 1-26), and $\nu_{N}^{\beta}$ satisfies a logarithmic Sobolev inequality. Associated concentration inequalities control the fluctuations of the periodic eigenvalues $\left(\lambda_{n}\right)$. For $\beta, N>0$ small, there exists a set of positive $\nu_{N}^{\beta}$ measure such that $\left( \pm \sqrt{2\left(\lambda_{2 n}+\lambda_{2 n-1}\right)}\right)_{n=0}^{\infty}$ gives a sampling sequence for Paley-Wiener space $P W(\pi)$ and the reproducing kernels give a Riesz basis. Let $\left(\mu_{j}\right)_{j=1}^{\infty}$ be the tied spectrum; then $\left(2 \sqrt{\mu_{j}}-j\right)$ belongs to a Hilbert cube in $\ell^{2}$ and is distributed according to a measure that satisfies Gaussian concentration for Lipschitz functions. The sampling sequence $\left(\sqrt{\mu_{j}}\right)_{j=1}^{\infty}$ arises from a divisor on the spectral curve, which is hyperelliptic of infinite genus. The linear statistics $\sum_{j} g\left(\sqrt{\lambda_{2 j}}\right)$ with test function $g \in P W(\pi)$ satisfy Gaussian concentration inequalities.

Keywords: Periodic eigenvalues, spectral theory, random operators, linear statistics Classification: 37L55

Résumé Cet article analyse le spectre périodique de l'équation de Schrödinger $-f^{\prime \prime}+q f=$ $\lambda f$ lorsque le potentiel est réel, périodique, aléatoire et muni de la mesure invariante $\nu_{N}^{\beta}$ de l'équation $\mathrm{KdV}$ périodique. Cette mesure $\nu_{N}^{\beta}$ est l'ensemble canonique modifié, donné par Bourgain (Comm. Math. Phys.166 (1994), 1-26), et $\nu_{N}^{\beta}$ satisfait une équation logarithmique de Sobolev. Des inégalités de concentration associées contrôlent les fluctuations des valeurs propres périodiques $\left(\lambda_{n}\right)$. Pour de petites valeurs $\beta, N>0$, il existe un ensemble de mesure $\nu_{N}^{\beta}$ positive tel que $\left( \pm \sqrt{2\left(\lambda_{2 n}+\lambda_{2 n-1}\right)}\right)_{n=0}^{\infty}$ donne une suite échantillonnage pour l'espace $P W(\pi)$ de Paley-Wiener et les noyaux reproduisants donnent une base de Riesz. Soit $\left(\mu_{j}\right)_{j=1}^{\infty}$ le spectre lié. Alors $\left(2 \sqrt{\mu_{j}}-j\right)$ appartient à un cube de Hilbert dans $\ell^{2}$ et il est distribué selon une mesure qui satisfait la concentration Gaussienne pour les fonctions de Lipschitz. La suite échantillonnage $\left(\sqrt{\mu_{j}}\right)_{j=1}^{\infty}$ découle d'un diviseur de la courbe spectrale, qui est hyperelliptique de genre infini. Les statistiques linéaires $\sum_{j} g\left(\sqrt{\lambda_{2 j}}\right)$ avec fonction test $g \in P W(\pi)$ satisfont les inégalités de concentration Gaussiennes. 


\section{Introduction}

A large class of systems can be modelled via differential equations of the form

$$
-f^{\prime \prime}(x)+q(x) f(x)=\lambda f(x) \quad(x \in \mathbf{R})
$$

where $q$ is a periodic potential function. Here $q: \mathbf{R} \rightarrow \mathbf{R}$ is a $2 \pi$-periodic and measurable function such that $N=\int_{0}^{2 \pi} q(x)^{2} d x /(2 \pi)$ is finite. Equation (1.1) is known variously as Hill's equation or the time-independent Schrödinger equation for potential scattering. By classical results, [20], Equation (1.1) admits an infinite increasing sequence of real eigenvalues

$$
\lambda_{0}<\lambda_{1} \leq \lambda_{2}<\lambda_{3} \leq \lambda_{4}<\lambda_{5} \leq \ldots
$$

each corresponding to either a nontrivial $2 \pi$-periodic solution giving the principal series of eigenvalues, or else an antiperiodic solution satisfying $f(x+2 \pi)=-f(x)$ giving the complementary series. The periodic spectrum $\left\{\lambda_{j}: j=0,1, \ldots\right\}$ partitions $\mathbf{R}$ into intervals of stability and instability for the nontrivial solutions for (1.1).

Hill's equation is closely related to the periodic Korteweg-de Vries (KdV) equation

$$
\frac{\partial u}{\partial t}+\frac{\partial^{3} u}{\partial x^{3}}+\beta u \frac{\partial u}{\partial x}=0
$$

Gardner, Greene, Kruskal and Miura [9] and Lax [17] noted that periodic spectrum of (1.1) is preserved if a time-dependent potential $q_{t}(x)$ evolves according to (1.3).

For suitable fixed $q$, much is known about the asymptotic behaviour of $\left\{\lambda_{j}\right\}$. Let

$$
\Omega_{N}=\left\{\phi \in L^{2}(\mathbf{T} ; \mathbf{R}): \int_{\mathbf{T}} \phi(x)^{2} \frac{d x}{2 \pi} \leq N\right\} \quad(N>0)
$$

It is known, for example, that if $q \in \Omega_{N}$ then both $\lambda_{2 j-1}$ and $\lambda_{2 j}$ are asymptotically

$$
\frac{j^{2}}{4}+\int_{0}^{2 \pi} q(x) \frac{d x}{2 \pi}+o(1) \quad(j \rightarrow \infty)
$$

and in particular that the $j^{\text {th }}$ intervals of instability $\left(\lambda_{2 j-1}, \lambda_{2 j}\right)$ has length $d_{j}=\lambda_{2 j}-\lambda_{2 j-1}$ such that $\left(d_{j}\right)_{j=1}^{\infty}$ forms an $\ell^{2}$ sequence; see [20] and [10]. Indeed the decay properties of the time-invariant sequence $\left\{d_{j}\right\}$ for a family of potentials solving (1.3) are closely related to regularity properties of those solutions. (See also [13].)

The central questions addressed in this paper concern the spectral properties of (1.1) for a random potential $q$. Given the link between (1.1) and (1.3) it is natural to choose $q$ according to the Gibbs measures $\nu_{N}^{\beta}$ for the periodic KdV system which were introduced by Bourgain [7]. These make $\left(\Omega_{N}, \nu_{N}^{\beta}\right)$ into an inner regular and Borel probability space. A typical $q$ in the support of $\nu_{N}^{\beta}$ is not differentiable, but by refining the classical spectral results from [20] 
we show that some, but not all, of the classical results apply. In [3], we proved concentration inequalities and logarithmic Sobolev inequalities for the measures $\nu_{N}^{\beta}$. The general principle is that a real Lipschitz function on $\Omega_{N}$ has very small average oscillation with respect to $\nu_{N}^{\beta}$; see [33, page 618]. For $q \in\left(\Omega_{N}, \nu_{N}^{\beta}\right)$, the sequence $\left(\lambda_{j}\right)$ is also random. Let $\mathcal{P}$ be the space of real periodic spectra. In Propositions 2.3 and 3.1, we show that $\mathcal{P}$ is embedded in a Hilbert cube.

We recall the definition of Hill's discriminant $\Delta$. Let $f_{\lambda}$ and $g_{\lambda}$ be the fundamental solutions of $(1.1)$ such that $f_{\lambda}(0)=1, f_{\lambda}^{\prime}(0)=0 ; g_{\lambda}(0)=0$ and $g_{\lambda}^{\prime}(0)=1$. Then

$$
\Delta(\lambda)=f_{\lambda}(2 \pi)+g_{\lambda}^{\prime}(2 \pi) \quad(\lambda \in \mathbf{C})
$$

defines an entire function of order $1 / 2$, and the periodic spectrum is the set of zeros of $4-\Delta(\lambda)^{2}$. The set $\left\{\lambda \in \mathbf{R}: \Delta(\lambda)^{2} \leq 4\right\}$ is typically an infinite union of closed and bounded intervals, called intervals of stability; whereas $\left\{\lambda \in \mathbf{R}: \Delta(\lambda)^{2}>4\right\}$ is typically an infinite union of open intervals $\left(\lambda_{2 j-1}, \lambda_{2 j}\right)$ such that all nontrivial solutions of (1.1) with $\lambda \in\left(\lambda_{2 j-1}, \lambda_{2 j}\right)$ are unbounded. Nevertheless, the tied eigenvalues of (1.1) for the boundary conditions $f(0)=$ $f(2 \pi)=0$ satisfy $\mu_{j} \in\left[\lambda_{2 j-1}, \lambda_{2 j}\right]$.

In section 4 we obtain concentration inequalities for the distribution of the tied eigenvalues $\mu_{j}$ and in section 5 for the periodic eigenvalues. Statistical information about the periodic spectrum $\left\{\lambda_{j}\right\}$ for random potentials is obtained by studying the distribution of scalar-valued random variables of the form $f\left(\left\{\lambda_{j}\right\}\right)$ for suitable functions $f$, and in particular, linear eigenvalue statistics. This study is analogous to the results on the statistical properties for the eigenvalues of random unitary $n \times n$ matrices taken from the compact group $U(n)$ under normalized Haar measure (see, for example, [12], or [29]). The proofs in sections 4 and 5 of the current paper use similar functional inequalities to their random matrix counterparts in [2].

Definition (i) (Linear Statistics) Let $(\Omega, \nu)$ be a probability space, and $t_{j}: \Omega \rightarrow \mathbf{R}$ random variables for $j \in \mathbf{Z}$. Let $H$ be a real reproducing kernel Hilbert space on $\mathbf{R}$, such that $h_{t} \in H$ satisfies $g(t)=\left\langle g, h_{t}\right\rangle_{H}$ for all $g \in H$ and $t \in \mathbf{R}$, and $t \mapsto\left\langle g, h_{t}\right\rangle$ is continuous. Then for all $g \in H$, there is a sequence of random variables $\left(g\left(t_{j}\right)\right)_{j=-\infty}^{\infty}$ on $(\Omega, \nu)$, and we define the corresponding linear statistics to be $\sum_{j=-m}^{m} g\left(t_{j}\right)$ or equivalently $\left\langle g, \sum_{j=-m}^{m} h_{t_{j}}\right\rangle$ for $m=0,1, \ldots$.

(ii) (Riesz basis) Let $\left(e_{j}\right)_{j=-\infty}^{\infty}$ be the usual orthonormal basis of $\ell^{2}$, and let $U: \ell^{2} \rightarrow H$ be a bounded linear operator with bounded inverse. Then $\left(U e_{j}\right)_{j=-\infty}^{\infty}$ is called a Riesz basis for $H$.

We choose $H$ to be independent of $q$, and then select the $\left(t_{j}\right)$ depending upon $q$. The mutual dependence of the $\left(t_{j}\right)$ is expressed in terms of the corresponding reproducing kernels $h_{t_{j}}$ and $\left[\left\langle h_{t_{j}}, h_{t_{k}}\right\rangle\right]$. We cannot expect that the $\left(h_{t_{j}}\right)$ will be an orthogonal sequence; nevertheless, 
we obtain conditions under which $\left(h_{t_{j}}\right)$ is a Riesz basis. This terminology is familiar from the theory of wavelets and sampling theory [25].

Definition (i) $(R P W(\pi))$ For $b>0$, the Paley-Wiener space $P W(b)$ is the complex Hilbert space of entire functions $f$ of exponential type such that

$$
\lim \sup _{y \rightarrow \pm \infty} \frac{\log |f(i y)|}{|y|} \leq b
$$

and $\int_{-\infty}^{\infty}|f(x)|^{2} d x$ finite; so that $P W(b)$ is a closed linear subspace of $L^{2}(\mathbf{R} ; \mathbf{C})$. Let $R P W(b)$ be the closed linear subspace of $L^{2}(\mathbf{R} ; \mathbf{R})$ consisting of those $g \in P W(b)$ such that $\overline{g(z)}=g(\bar{z})$.

(ii) (sinc) The normalised cardinal sine function is $\operatorname{sinc}(x)=(\sin \pi x) /(\pi x)$, so that sinc $\in$ $R P W(\pi)$. Moreover, $\operatorname{sinc}(t-s)$ is the reproducing kernel for $s \in \mathbf{R}$ and $P W(\pi)$ as in [26], so

$$
g(s)=\int_{-\infty}^{\infty} g(t) \operatorname{sinc}(t-s) d t \quad(g \in P W(\pi))
$$

(iii) (Sampling points) Introduce the points $\left(t_{n}\right)_{n=-\infty}^{\infty}$ by

$$
t_{n}= \begin{cases}\sqrt{\lambda_{2 n}-\lambda_{0}}, & n=1,2, \ldots ; \\ 0, & n=0 ; \\ -\sqrt{\lambda_{-2 n}-\lambda_{0}}, & n=-1,-2, \ldots\end{cases}
$$

Shannon's sampling theorem refers to sampling on $Z=(j)_{j=-\infty}^{\infty}$. In the current paper, we sample on $\left(t_{j}\right)_{J=-\infty}^{\infty}$, where $t_{j}$ are random variables on $\left(\Omega_{N}, \nu_{N}^{\beta}\right)$. We can view the differences $\left(\xi_{j}=t_{j}-j\right)_{j=-\infty}^{\infty}$ as random jitters in the sampling points. For the linear statistics associated with the $\mathrm{KdV}$ measure, in section 5 we prove a concentration inequality which shows that the linear statistic $\sum_{j=-m}^{m} g\left(t_{j}\right)$ for $g \in R P W(\pi)$ is tightly concentrated about its mean value with Gaussian decay away from the mean. For typical $g \in R P W(\pi)$, the series $\sum_{n=-\infty}^{\infty} g(n)$ is not absolutely convergent, so we use the normalized series $\sum_{j=-\infty}^{\infty}\left(g\left(t_{j}\right)-g(j)\right)$ and its partial sums. The mutual dependence of the $\lambda_{j}$ is described in terms of the Gram matrix, and its generalized determinant, which is defined as follows.

Definition $\left(\operatorname{det}_{2}\right)$ Let $A$ be a Hilbert-Schmidt operator with norm $\|A\|_{H S}$ and eigenvalues $\left(\kappa_{j}\right)_{j=0}^{\infty}$ listed according to multiplicity. Then the Carleman determinant of $I+A$ is defined by the convergent infinite product

$$
\operatorname{det}_{2}(I+A)=\prod_{j=0}^{\infty}\left(I+\kappa_{j}\right) e^{-\kappa_{j}}
$$

We write $I+H S=\left\{T \in B\left(\ell^{2}\right): T-I \in H S\right\}$ and observe that $\mathcal{G}=\left\{T \in I+H S: \operatorname{det}_{2} T \neq 0\right\}$ is an infinite dimensional multiplicative group. 
Given $t=\left(t_{j}\right)$ as in (1.9) let $h_{t_{j}}(s)=\operatorname{sinc}\left(s-t_{j}\right)$. Then the Gram matrix, defined by $G(t)=\left[\operatorname{sinc}\left(t_{j}-t_{k}\right)\right]$, determines the properties of $\left(h_{t_{j}}\right)_{j=-\infty}^{\infty}$ up to unitary equivalence. In section 6 , we show that $G(t) \in \mathcal{G}$ where $t=t(q)$ and $q$ belongs to a subset of $\Omega_{N}$ of positive $\nu_{N}^{\beta}$ measure, and we use this fact to show that $\left(h_{t_{j}}\right)_{j=-\infty}^{\infty}$ is a Riesz basis for $R P W(\pi)$. In section 7 , we interpret linear statistics as divisors on the spectral curves.

Hill's curve is the transcendental hyperelliptic curve

$$
\mathcal{E}=\left\{(z, \lambda) \in \mathbf{C}^{2}: z^{2}=4-\Delta(\lambda)^{2}\right\}
$$

which has branch points at the periodic eigenvalues. Choosing $\mu_{j} \in\left(\lambda_{2 j-1}, \lambda_{2 j}\right)$, we introduce a divisor $\delta$ and a differential form $\omega_{\infty}$ such that

$$
\omega_{\infty}(\delta)=\sum_{j} \int_{\mu_{j}}^{\lambda_{2 j}} \frac{\Delta^{\prime}(\lambda) d \lambda}{\sqrt{\Delta(\lambda)^{2}-4}} .
$$

This formula requires careful interpretation in the case of random $q$. In section 7 , we show that the curve $\mathcal{E}$ has a real Jacobian $\mathbf{X}$, which is the real part of an infinite dimensional complex torus. In Proposition 7.2, we produce a map $\Omega_{N} \rightarrow \mathbf{X} \times \mathcal{P}$ which is one-to-one, up to translating the potential.

Analogously, a compact and connected Lie group has a maximal torus $T$ and set of cosets $G / T$ with fibration $T \times(G / T) \rightarrow G$. In the group $U(n)$ of complex unitary $n \times n$ matrices, the maximal torus $\mathbf{T}^{n}$ is realised as the space of diagonal unitary matrices. The eigenvalue map $U \mapsto \operatorname{Diag}\left(e^{i \theta_{j}}\right)$ induces a probability measure $\mu_{W}$ on $\mathbf{T}^{n}$ from Haar measure on $U(n)$, as expressed by the Weyl denominator formula [14].

The table below expresses the analogy between linear statistics for the KdV measure and linear statistics for $U(n)$. Let $f \in L^{1}(\mathbf{T} ; \mathbf{C})$ have Fourier coefficients $\hat{f}_{j}$, and form the Toeplitz determinant $D_{n}(f)=\operatorname{det}\left[\hat{f}_{j-k}\right]_{j, k=0}^{n-1}$. In particular, suppose that $g \in L^{1}(\mathbf{T} ; \mathbf{C})$ belongs to $H^{1 / 2}(\mathbf{T})$ so that $\sum_{k \in \mathbf{Z}}|k||\hat{g}(k)|^{2}$ finite and let $f=e^{g}$. Then Johansson [12] proved a strong Szegö theorem, which describes the asymptotic growth of $D_{n}\left(e^{g}\right)$ as $n \rightarrow \infty$ in terms of a quadratic form in $g$, and established a central limit theorem for the linear statistics. Our concentration results are quite analogous.

$$
\begin{array}{ccc} 
& \text { Hill's curve } & \text { Unitary group } \\
\text { Probability space } & \Omega_{N}=\left\{q \in L^{2}(\mathbf{T}) ;\|q\|_{2}^{2} \leq N\right\} & U(n) \\
\text { Probability measure } & \nu_{N}^{\beta} & \mu_{W} \\
\text { Eigenvalues } & \mathbf{X}=\left\{\left(\mu_{j}\right)_{j=1}^{\infty}\right\} & \mathbf{T}^{n}=\left\{\left(e^{i \theta_{j}}\right)_{j=1}^{n}\right\} \\
\text { Test functions } & g(z) \in R P W(\pi) & g \in H^{1 / 2}(\mathbf{T}) \\
\text { Linear statistic } & \sum_{j=-m}^{m}\left(g\left(t_{j}\right)-g(j)\right) & \sum_{j=1}^{n} g\left(e^{i \theta_{j}}\right)-n \int_{\mathbf{T}} g\left(e^{i \theta}\right) \frac{d \theta}{2 \pi} \\
\text { Quadratic form } & 2^{-1} \sum_{j=-m}^{m}\left|g^{\prime}\left(t_{j}\right)\right|^{2} & \iint_{\mathbf{T}^{2}}-\log \left|e^{i \theta}-e^{i \phi}\right| g^{\prime}\left(e^{i \theta}\right) g^{\prime}\left(e^{i \phi}\right) \frac{d \theta}{2 \pi} \frac{d \phi}{2 \pi}
\end{array}
$$




\section{Concentration of measures}

In this section we prove some concentration inequalities for three measures. We introduce the infinite dimensional phase space of $u: \mathbf{T} \times(0, \infty) \rightarrow \mathbf{R}$ such that $u(\cdot, t) \in L^{2}(\mathbf{T})$ for all $t>0$, and the densely-defined Hamiltonian

$$
H(u)=\frac{1}{2} \int_{\mathbf{T}}\left(\frac{\partial u}{\partial x}(x, t)\right)^{2} \frac{d x}{2 \pi}-\frac{\beta}{6} \int_{\mathbf{T}} u(x, t)^{3} \frac{d x}{2 \pi},
$$

where $\beta>0$ is the inverse temperature. The canonical equation of motion reduces to the $\mathrm{KdV}$ equation (1.3) which gives a nonlinear evolution on $L^{2}(\mathbf{T})$ such that $H(u)$ and $\int_{\mathbf{T}} u(x, t)^{2} \frac{d x}{2 \pi}$ are invariant with respect to $t$. The relevance to (1.1) is that the flow generated by the $\mathrm{KdV}$ equation $\frac{\partial q}{\partial t}=3 q \frac{\partial q}{\partial x}-\frac{1}{2} \frac{\partial^{3} q}{\partial x^{3}}$ preserves the periodic spectrum.

For some normalizing constant $Z_{N}(\beta)$, there exists a probability measure

$$
\nu_{N}^{\beta}(d \phi)=Z_{N}(\beta)^{-1} \mathbf{I}_{\Omega_{N}}(\phi) e^{-H(\phi)} \prod_{e^{i x} \in \mathbf{T}} d \phi(x)
$$

which is Radon, in the sense of being inner-regular and defined on the $\sigma$-algebra generated by the Borel sets in $L^{2}$. Bourgain [7] constructed this probability measure using random Fourier series, as follows. Let $\left(\gamma_{n}\right)_{n=-\infty}^{\infty}$ be mutually independent standard Gaussian random variables on some probability space $(\Omega, \mathbf{P})$, and let $\gamma$ be the Gaussian probability measure on $L^{2}(\mathbf{T} ; \mathbf{R})$ that is induced by the map

$$
\omega \mapsto \phi_{\omega}(x)=\gamma_{0}+\sum_{n=-\infty}^{-1} \gamma_{n} \frac{\sin n x}{n}+\sum_{n=1}^{\infty} \gamma_{n} \frac{\cos n x}{n}
$$

This gives an interpretation to the product factor in $(2.2)$.

Let the Fourier expansion of the potential be $q(x)=(1 / 2) a_{0}+\sum_{n=1}^{\infty}\left(a_{n} \cos n x+b_{n} \sin n x\right)$, where the Fourier coefficients are real random variables on $\left(\Omega_{N}, \nu_{N}^{\beta}\right)$ satisfying $\left(a_{0} / 2\right)^{2}+$ $2^{-1} \sum_{n=1}^{\infty}\left(a_{n}^{2}+b_{n}^{2}\right) \leq N$.

Lemma 2.1 Let $A=\left[\alpha_{j k}\right]$ be a trace class and real symmetric matrix on $\ell^{2}$ with eigenvalues $\left(\sigma_{j}\right)_{j=0}^{\infty}$ which are listed according to multiplicity, and such that $\left(\left|\sigma_{j}\right|\right)_{j=0}^{\infty}$ is decreasing, and let $\gamma_{j}$ be mutually independent normalized Gaussian random variables on $L^{2}$. Then for $0<$ $\varepsilon<1 / 2$ and $K=\pi / 2 \sqrt{1-2 \varepsilon}$, the random variable $Q=\sum_{j, k} \alpha_{j k} \gamma_{j} \gamma_{k}$ satisfies

$$
\int_{\Omega_{N}} e^{s Q} \nu_{N}^{0}(d q) \leq\left(1-e^{-\varepsilon N} K\right)^{-1} \prod_{j=0}^{\infty}\left(1-2 s \sigma_{j}\right)^{-1 / 2} \quad\left(N>\varepsilon^{-1} \log K, \quad 2 s\left|\sigma_{0}\right|<1\right)
$$

Proof. The sequence $\left(\left|\sigma_{j}\right|\right)_{j=0}^{\infty}$ gives the eigenvalues of the positive square root $\left(A^{2}\right)^{1 / 2}$, hence gives a summable sequence, so the product on the right-hand side is absolutely convergent. 
There exists a real orthogonal matrix on $\ell^{2}$ such that $U^{\dagger} A U$ is the real diagonal matrix $\operatorname{diag}\left(\sigma_{j}\right)_{j=0}^{\infty}$.

By elementary results regarding Gaussian random variables, we can compute

$$
\int_{L^{2}} e^{s Q} \gamma(d q)=\prod_{j=0}^{\infty} \int_{-\infty}^{\infty} e^{s \sigma_{j}-x^{2} / 2} \frac{d x}{\sqrt{2 \pi}}=\prod_{j=0}^{\infty}\left(1-2 s \sigma_{j}\right)^{-1 / 2}
$$

When $\sigma_{j}=1 / j^{2}$ we can compute this product explicitly, and so for $0<\varepsilon<1 / 2$ we obtain

$$
\int_{L^{2}} e^{\varepsilon\|q\|^{2}} \gamma(d q)=\frac{1}{\sqrt{1-2 \varepsilon}} \frac{\sqrt{\varepsilon \pi}}{\sin \sqrt{\varepsilon \pi}}=K_{\varepsilon}
$$

so by Chebyshev's inequality, $\gamma\left(\Omega_{N}^{c}\right) \leq K_{\varepsilon} e^{-\varepsilon N}$; hence the normalizing constant for $\nu_{N}^{0}$ on $\Omega_{N}$ satisfies

$$
\int_{\Omega_{N}} \gamma(d q)>\max \left\{1-K_{\varepsilon} e^{-\varepsilon N}, 0\right\}
$$

The following transportation cost bounds the distance between $\nu_{N}^{0}$ and $\nu_{N}^{\beta}$ in the Wasserstein metric; see [32] for a general discussion.

Lemma 2.2 For all $\beta, N>0$, there exists $\kappa(\beta, N)$ such that if $f: \Omega_{N} \rightarrow \mathbf{R}$ is any Lipschitz function such that $|f(p)-f(q)| \leq L\|p-q\|_{L^{2}}$ for all $p, q \in \Omega_{N}$, then

$$
\left|\int_{\Omega_{N}} f(q) \nu_{N}^{0}(d q)-\int_{\Omega_{N}} f(q) \nu_{N}^{\beta}(d q)\right| \leq L \beta N \kappa
$$

$A$ valid choice of $\kappa$ is $\kappa=e^{C \beta^{5 / 2} N^{9 / 4}}$ where $C$ is some absolute constant taken from [3].

Proof. By the Kantorovich-Rubinstein duality theorem [32, p 34], the quantity (2.8) in the Lemma may be expressed as

$$
\left|\int_{\Omega_{N}} f(q) \nu_{N}^{0}(d q)-\int_{\Omega_{N}} f(q) \nu_{N}^{\beta}(d q)\right|=L \inf _{\pi}\left\{\iint_{\Omega_{N} \times \Omega_{N}}\|p-q\|_{L^{2}} \pi(d p d q)\right\}
$$

where the infimum is taken over all the Radon probability measures on $\Omega_{N} \times \Omega_{N}$ that have marginals $\nu_{N}^{0}$ and $\nu_{N}^{\beta}$. The infimum on the right-hand side of (2.9) defines the transportation cost for the cost function $\|p-q\|_{L^{2}}$.

Next we note that $\nu_{N}^{0}$ is symmetrical in distribution with respect to $a_{n} \leftrightarrow-a_{n}$ and $b_{n} \leftrightarrow$ $-b_{n}$ so some expectations are easy to compute. Note also that $F(q)=-\beta \int_{\mathbf{T}} q(x)^{3} d x /(2 \pi)$ is Lipschitz on $\Omega_{N}$ with $L=\beta N$, and $\int_{\Omega_{N}} F(q) \nu_{N}^{0}(d q)=0$. 
We bound the left-hand side of (2.9) in terms of the relative entropy, where the relative entropy of $\nu_{N}^{0}$ with respect to $\nu_{N}^{\beta}$ satisfies

$$
\begin{aligned}
\operatorname{Ent}\left(\nu_{N}^{0} \mid \nu_{N}^{\beta}\right) & =\int_{\Omega_{N}} \log \frac{d \nu_{N}^{0}}{d \nu_{N}^{\beta}} d \nu_{N}^{0} \\
& =\int_{\Omega_{N}}\left(-F(q)+\log Z_{N}(\beta)\right) \nu_{N}^{0}(d q) \\
& =\log Z_{N}(\beta) .
\end{aligned}
$$

By the concentration inequality for Gaussians, the normalizing constant for $\nu_{N}^{\beta}$ satisfies

$$
Z_{N}^{\beta}=\int_{\Omega_{N}} e^{F(q)} \nu_{N}^{0}(d q) \leq e^{\beta^{2} N^{2}}
$$

The concentration inequality for $\nu_{N}^{\beta}$ which was proved in [3] gives the upper bound

$$
(2.9) \leq L \kappa\left(2 \operatorname{Ent}\left(\nu_{N}^{0} \mid \nu_{N}^{\beta}\right)\right)^{1 / 2}
$$

Hence by $(2.11)$ and $(2.12)$ we have the bound $(2.9) \leq \sqrt{2} L \kappa \beta N$. This estimate improves as $\beta \rightarrow 0$, since $\nu_{N}^{\beta}$ converges in Wasserstein metric to $\nu_{N}^{0}$.

Proposition 2.3 (i) There exists $N_{1}>0$ such that for $0<N<N_{1}$ there exist positive constants $c(N, \beta)$ and $C(N, \beta)$ such that the mean length of the $j^{\text {th }}$ interval of instability satisfies

$$
\frac{c(N, \beta)}{j} \leq \int_{\Omega_{N}}\left(\lambda_{2 j}-\lambda_{2 j-1}\right) \nu_{N}^{\beta}(d q) \leq \frac{C(N, \beta)}{j} \quad(j=1,2, \ldots) .
$$

(ii) With probability one with respect to $\nu_{N}^{\beta}$, all the periodic eigenvalues are simple, so $\lambda_{j}<$ $\lambda_{j+1}$ for all $j$.

Proof. (i) Erdelyi [8] showed that there exists $N_{1}$ such that for all $q \in \Omega_{N}$ with $0<N<N_{1}$, the length of the $j^{\text {th }}$ spectral gap is equivalent to the $j^{\text {th }}$ Fourier coefficient of $q$, so

$$
\lambda_{2 j}-\lambda_{2 j-1} \asymp c N\left|\int_{0}^{2 \pi} q(x) e^{-i j x} \frac{d x}{2 \pi}\right| \quad(j \rightarrow \infty) .
$$

From (2.3), we deduce that

$$
\int_{\Omega_{N}}\left(\lambda_{2 j}-\lambda_{2 j-1}\right)^{2} \nu_{N}^{0}(d q) \leq \frac{C N^{2}}{j^{2}} \int_{\Omega_{N}} \gamma_{j}^{2} \nu_{N}^{0}(d q),
$$

and so we can use the Cauchy-Schwarz inequality to obtain an upper bound involving the measure $\nu_{N}^{\beta}$, namely

$$
\begin{aligned}
\left(\int_{\Omega_{N}}\left(\lambda_{2 j}-\lambda_{2 j-1}\right) \nu_{N}^{\beta}(d q)\right)^{2} & \leq \int_{\Omega_{N}}\left(\lambda_{2 j}-\lambda_{2 j-1}\right)^{2} \nu_{N}^{0}(d q) \int_{\Omega_{N}}\left(\frac{d \nu_{N}^{\beta}}{d \nu_{N}^{0}}\right)^{2} \nu_{N}^{0}(d q) \\
& \leq \frac{C(N, \beta)}{j^{2}}
\end{aligned}
$$


where the constants involve the normalizing constant for the Gibbs measure.

To prove the lower bound in (2.13), we introduce the events $A_{N}(j)=\left\{\omega:|\hat{q}(j)|^{2} \leq\right.$ $N / 2\}$ and $B_{N}(j)=\left\{\omega: \sum_{k: k \neq \pm j}|q|^{2} \leq N / 2\right\}$ which are independent in $\left(L^{2}, \gamma\right)$ and satisfy $A_{N}(j) \cap B_{N}(j) \subseteq \Omega_{N}$. Hence there exists $c_{N}>0$ such that

$$
\begin{aligned}
\frac{c_{N}}{|j|^{1 / 2}} & \leq \int_{A_{N}(j)}|\hat{q}(j)|^{1 / 2} d \gamma \int_{B_{N}(j)}\left(\sum_{k: k \neq \pm j}|\hat{q}(k)|^{2}\right)^{1 / 4} d \gamma \\
& \leq \int_{\Omega_{N}}|\hat{q}(j)|^{1 / 2}\left(\sum_{k}|\hat{q}(k)|^{2}\right)^{1 / 4} d \gamma
\end{aligned}
$$

wherein $\left(\sum_{j=-\infty}^{\infty}|\hat{q}(j)|^{2}\right)^{1 / 4} \leq N^{1 / 4}$. Now by (2.3) and (2.14) we have

$$
\begin{aligned}
(2.17) & \leq Z_{N}(\beta) \int_{\Omega_{N}} \sqrt{\lambda_{2 j}-\lambda_{2 j-1}} d \nu_{N}^{0} \\
& \leq Z_{N}(\beta)\left(\int_{\Omega_{N}}\left(\lambda_{2 j}-\lambda_{2 j-1}\right) d \nu_{N}^{\beta}\right)^{1 / 2}\left(\int_{\Omega_{N}}\left(\frac{d \nu_{N}^{0}}{d \nu_{N}^{\beta}}\right)^{2} d \nu_{N}^{0}\right)^{1 / 2} .
\end{aligned}
$$

(ii) It suffices to show that $\sum_{j=1}^{\infty} j^{-2}\left|\log \left(\lambda_{2 j}-\lambda_{2 j-1}\right)\right|$ is integrable with respect to $\nu_{N}^{\beta}$. The required estimate is (2.16), with $\left|\log \left(\lambda_{2 j}-\lambda_{2 j-1}\right)\right|$ replacing $\lambda_{2 j}-\lambda_{2 j-1}$.

Remark 2.4 An unwelcome consequence of (2.13) is that $\sum_{j=1}^{\infty}\left(\lambda_{2 j}-\lambda_{2 j-1}\right) \lambda_{2 j}^{k}$ diverges for $k \geq 0$ with probability one, so the polynomial approximation arguments from section 10 of [22] become inapplicable. In Proposition 7.2, we consider the inverse spectral problem for $q \in\left(\Omega_{N}, \nu_{N}^{\beta}\right)$.

\section{Fluctuations and sampling of the periodic eigenvalues}

In this section we analyse the discriminant of (1.6) and obtain detailed information about the fluctuations of the periodic eigenvalues by using the concentration results of section 2 . Loosely speaking, we show that the intervals of instability $\left[\lambda_{2 j-1}, \lambda_{2 j}\right]$ are random with mean length of order $1 / j$ and centre of order $j^{2} / 4$. First we obtain a version of Borg's estimates from [20].

Proposition 3.1 For all $q \in \Omega_{N}$ and all $\delta>0$, the periodic eigenvalues of (1.1) satisfy

$$
\left|\sqrt{\lambda_{2 n}}-\frac{n}{2}\right| \leq \frac{\sqrt{\beta N}}{n^{1-\delta}} \quad\left(n>(\beta N)^{1 / \delta} \kappa^{1 / \delta}\right)
$$

so $\left\{\left(\sqrt{\lambda_{4 n}-\lambda_{0}}-n\right)_{n=1}^{\infty}: q \in \Omega_{N}\right\}$ is relatively compact in the norm topology of $L^{2}$.

Proof. For $0<\eta \leq 2 \pi$, let $V, W: L^{\infty}[0, \eta] \rightarrow L^{\infty}[0, \eta]$, where $V=V(q)$ and $W=W(q)$, be the bounded linear operators

$$
V f(x)=\int_{0}^{x} \cos (\sqrt{\lambda}(x-t)) q(t) f(t) d t \quad\left(f \in L^{\infty}[0, \eta]\right)
$$




$$
W g(x)=\int_{0}^{x} \sin (\sqrt{\lambda}(x-t)) q(t) g(t) d t \quad\left(g \in L^{\infty}[0, \eta]\right) .
$$

These integral operators have operator norms that satisfy

$$
\|V\|_{o p},\|W\|_{o p} \leq \cosh (\eta Y) \int_{0}^{\eta}|q(t)| d t \leq \cosh (\eta Y) \sqrt{\eta N}
$$

for all $q \in \Omega_{N}$. This estimate is uniform over the probability space $\Omega_{N}$ and not random, and enables us to obtain non random error terms in the following.

The fundamental solutions of Hill's equation satisfy

$$
\begin{aligned}
& f_{\lambda}(x)=\cos \sqrt{\lambda} x+\frac{1}{\sqrt{\lambda}} W f_{\lambda}(x), \\
& g_{\lambda}(x)=\frac{\sin \sqrt{\lambda} x}{\sqrt{\lambda}}+\frac{1}{\sqrt{\lambda}} W g_{\lambda}(x)
\end{aligned}
$$

so the discriminant may be expressed as the series

$$
\begin{aligned}
\Delta(\lambda) & =2 \cos 2 \sqrt{\lambda} \pi+\frac{1}{\sqrt{\lambda}} \int_{0}^{2 \pi}(q(t)+q(2 \pi-t)) \sin \sqrt{\lambda}(2 \pi-t) \cos \sqrt{\lambda} t d t \\
& +\frac{1}{\lambda}\left(W^{2}(\cos \sqrt{\lambda} t)(2 \pi)+V W(\sin \sqrt{\lambda} t)(2 \pi)\right) \\
& +\frac{1}{\lambda^{3 / 2}}\left(W^{3}(\cos \sqrt{\lambda} t)(2 \pi)+V W^{2}(\sin \sqrt{\lambda} t)(2 \pi)\right)+O\left(\frac{1}{\lambda^{2}}\right) ;
\end{aligned}
$$

in which the coefficient of $\lambda^{-k / 2}$ consists of $2^{k}$ terms, each of which is a product of $k$ factors of $V$ and $W$; hence the series converges for $\lambda>4 N$. We consider the terms on the right hand side of (3.7) in turn: $2 \cos 2 \sqrt{\lambda} \pi$ is the main term and is independent of $q$; the next term is random, and reduces by Fourier analysis to $\left(\pi a_{0} \sin 2 \sqrt{\lambda} \pi\right) / \sqrt{\lambda}$; then the coefficient of $1 / \lambda$ is

$$
Q=\int_{0}^{2 \pi} q(t) \int_{0}^{t} \sin \sqrt{\lambda}(t-x) \sin \sqrt{\lambda}(2 \pi-t+x) q(x) d x d t,
$$

which is a real quadratic form in $q$, and hence may be expressed as the symmetrical expression involving the symmetric kernel

$$
K(x, y)= \begin{cases}\sin \sqrt{\lambda}(y-x) \sin \sqrt{\lambda}(2 \pi-y+x) & \text { for } 0 \leq x \leq y \leq 2 \pi \\ \sin \sqrt{\lambda}(x-y) \sin \sqrt{\lambda}(2 \pi-x+y) & \text { for } 0 \leq y \leq x \leq 2 \pi\end{cases}
$$

this defines a trace class linear operator on $L^{2}[0,2 \pi]$, as one checks by considering the operation of $K$ on $\left(e^{i n x}\right)_{n=-\infty}^{\infty}$. Passing to the Fourier coefficients, we deduce that

$$
Q=\frac{1}{8} a_{0}^{2}\left(\frac{\pi \sin 2 \pi \sqrt{\lambda}}{\sqrt{\lambda}}-2 \pi^{2} \cos 2 \pi \sqrt{\lambda}\right)-\pi \sqrt{\lambda} \sin 2 \pi \sqrt{\lambda} \sum_{n=1}^{\infty} \frac{a_{n}^{2}+b_{n}^{2}}{n^{2}-4 \lambda} .
$$


Hence the discriminant satisfies

$$
2-\Delta(\lambda)=4 \sin ^{2} \pi \sqrt{\lambda}-\frac{2 \pi a_{0} \sin \pi \sqrt{\lambda}}{\sqrt{\lambda}} \cos \pi \sqrt{\lambda}+O\left(\frac{\beta N}{\lambda}\right)
$$

for large $\lambda$. From the product formula for sin, the factors are holomorphic functions for $\Re \lambda>1$. We let $\sqrt{\lambda}=2 n+\tau+i \sigma$ where $|\tau+i \sigma|=\sqrt{\beta N} / n^{1-\delta}$ and apply Rouché's theorem to compare $2-\Delta(\lambda)$ with $4 \sin ^{2} \pi \sqrt{\lambda}$. Note that $\sin ^{2} \pi \sqrt{\lambda}$ has a double zero at $\lambda=n^{2}$, and

$$
\left|4 \sin ^{2} \pi \sqrt{\lambda}\right|=4 \sin ^{2} \pi \tau+4 \sinh ^{2} \pi \sigma>4\left(\tau^{2}+\sigma^{2}\right),
$$

which is greater than $\beta N /|\lambda|$. This gives a pair of zeros of $2-\Delta(\lambda)$, namely eigenvalues $\lambda_{4 n-1}$ and $\lambda_{4 n}$ of the principal series.

In the analysis of periodic spectra in [20] page 24, the authors assume that $q$ has mean zero. In our case, however, the value of $a_{0}(q)$ is random, we now consider its influence on the spectrum. We have a coarse estimate on $a_{0}$, namely $\left|a_{0}\right| \leq \sqrt{N}$, so

$$
\left|\frac{\pi a_{0}}{\sqrt{\lambda}}\right| \leq \frac{\pi \sqrt{N}}{2 n} \leq \frac{\pi}{n^{\delta} \sqrt{\beta}}|\tau+i \sigma| .
$$

which is an appropriate estimate when $\beta>0$ is not small.

Also, note that $a_{0}=\int_{0}^{2 \pi} q(x) d x / \pi$ is a Lipschitz function of $q \in \Omega_{N}$ with $L=1$, so by Lemma 2.2, we have

$$
\left|\int_{\Omega_{N}} a_{0}(q) \nu_{N}^{\beta}(d q)-\int_{\Omega_{N}} a_{0}(q) \nu_{N}^{0}(d q)\right| \leq N \beta \kappa
$$

where $\kappa=e^{C \beta^{5 / 2} N^{9 / 4}}$; so this estimate is advantageous when $\beta>0$ is small. Then by the concentration inequality Corollary 2 of [3], we have

$$
\nu_{N}^{\beta}\left\{q \in \Omega_{N}:\left|a_{0}(q)-\int a_{0} d \nu_{N}^{\beta}\right|>t\right\} \leq 2 e^{-t^{2} / 4 \kappa}
$$

and by choosing $0<\varepsilon<\delta$ and $t=2 \beta \sqrt{\kappa} n^{\varepsilon}$ we deduce that

$$
\nu_{N}^{\beta}\left\{q \in \Omega_{N}:\left|a_{0}(q)-\int a_{0} d \nu_{N}^{\beta}\right|>2 \beta \sqrt{\kappa} n^{\varepsilon}\right\} \leq 2 e^{-\beta n^{\varepsilon}} .
$$

By the Borel-Cantelli Lemma, we deduce that

$$
\nu_{N}^{\beta}\left\{q \in \Omega_{N}:\left|a_{0}(q)\right| \leq \beta N \kappa+2 \beta \sqrt{\kappa} n^{\varepsilon} \text { for all but finitely many } n\right\}=1 \text {. }
$$

We deduce that, on a set of full $\nu_{N}^{\beta}$ measure, the inequality

$$
\frac{\pi\left|a_{0}\right|}{\sqrt{|\lambda|}} \leq\left(\frac{\kappa \sqrt{\beta N}}{n^{\delta}}+\frac{2 \sqrt{\beta} \kappa}{n^{\delta-\varepsilon}}\right)|\tau+i \sigma|
$$


holds for all but finitely many $n$.

It follows that on the curve $\mathcal{C}_{n}$ defined by $\sqrt{\lambda}=2 n+\tau+i \sigma$, we have

$$
\left|4 \sin ^{2} \pi \sqrt{\lambda}\right|>\left|(2-\Delta(\lambda))-4 \sin ^{2} \pi \sqrt{\lambda}\right|
$$

so that $2-\Delta(\lambda)$ and $4 \sin ^{2} \pi \sqrt{\lambda}$ both have two zeros inside $\mathcal{C}_{n}$, and by the basic theory of Hill's equation, the zeros are real. Likewise, the discriminant satisfies

$$
2+\Delta(\lambda)=4 \cos ^{2} \pi \sqrt{\lambda}+\frac{2 \pi a_{0} \sin \pi \sqrt{\lambda}}{\sqrt{\lambda}} \cos \pi \sqrt{\lambda}+O\left(\frac{\beta N}{|\lambda|}\right) .
$$

hence $2+\Delta(\lambda)$ has a pair of real zeros $\lambda_{4 n-3}$ and $\lambda_{4 n-2}$ of the complementary series when $\sqrt{\lambda}$ is close to $(2 n+1) / 2$.

Finally, observe that for all $C>0$ and $0<\delta<1 / 2$, the subset $\left\{\left(\xi_{n}\right)_{n=1}^{\infty}:\left|\xi_{n}\right| \leq\right.$ $\left.C / n^{1-\delta}, n=1,2, \ldots\right\}$ of $\ell^{2}$ is a compact Hilbert cube.

Definition (Cartwright class) The Cartwright class [15, 24] is the space of the entire functions $f$ such that

$$
\int_{-\infty}^{\infty} \frac{\log _{+}|f(x)|}{1+x^{2}} d x<\infty .
$$

Proposition 3.2 For $q \in\left(\Omega_{N}, \nu_{N}^{\beta}\right)$, let $\left(t_{j}\right)_{j=-\infty}^{\infty}$ be as in (1.9), where the $\lambda_{j}$ are the periodic eigenvalues of (1.1). Then for all $q$ in a subset of $\Omega_{N}$ with measure one with respect to $\nu_{N}^{\beta}$ there exists $b_{0}>0$ such that, if $f$ is an entire functions of exponential type $b<b_{0}$ and

$$
\sum_{j \in \mathbf{Z}} \frac{\log _{+}\left|f\left(t_{j}\right)\right|}{1+t_{j}^{2}}<\infty
$$

then $f$ belongs to the Cartwright class.

Proof. In his study of almost periodic functions, Bohr called a discrete subset $\Lambda$ of $\mathbf{R}$ relatively dense if there exists $L>0$ such that $\Lambda$ intersects all intervals $[a, a+L]$ for $a \in \mathbf{R}$. Pedersen [27] refined this, by saying that a discrete subset $T$ of $\mathbf{R}$ is $h$-dense if there exists $h>0$ such that, outside of some bounded set, $T$ intersects all bounded intervals of length $h$. By Proposition 3.1 , the set $T=\left\{t_{j}: j \in \mathbf{Z}\right\}$ is $3 / 2$ dense, symmetric and separated, so that there exists $\delta>0$, depending on $q$, such that $\left|t_{k}-t_{m}\right| \geq \delta$ for all $k \neq m$. Hence by Theorem 5.1 of [27], all entire functions $f$ of exponential type $b$ for $0 \leq b \leq b_{0}$ and such that (3.20) holds also have finite logarithmic integral (3.19), hence belong to Cartwright's class.

The connection between the logarithmic integral and exponential sums is subtle, so we refer the reader to [15]. We summarize some standard results regarding (1) sampling and (2) interpolation for the Paley-Wiener space. Suppose that $t=\left(t_{j}\right)_{j=-\infty}^{\infty}$ is separated (uniformly discrete in [26, p 219]), so that there exists $\delta>0$ such that $\left|t_{k}-t_{m}\right| \geq \delta$ for all $k \neq m$. Then 
the sampling map $S_{t}: P W(\pi) \rightarrow \ell^{2}: f \mapsto\left(f\left(t_{j}\right)\right)_{j=-\infty}^{\infty}$ defines a bounded linear operator, hence the adjoint map is also bounded, where

$$
S_{t}^{\dagger}: \ell^{2} \rightarrow P W(\pi): \quad S_{t}^{\dagger}\left(a_{j}\right)=\sum_{j=-\infty}^{\infty} a_{j} \operatorname{sinc}\left(s-t_{j}\right)
$$

(1) If $S_{t}$ is an embedding, so there exists $B>0$ such that $\left\|S_{t} f\right\| \geq B\|f\|$ for all $f \in P W(\pi)$, then $\left(\operatorname{sinc}\left(s-t_{j}\right)\right)_{j=-\infty}^{\infty}$ is a frame in $P W(\pi)$, and we say that $\left(t_{j}\right)$ is a sampling sequence.

(2) If $S_{t}^{\dagger}$ is an embedding, or equivalently $S_{t}: P W(\pi) \rightarrow \ell^{2}$ is surjective, then $(\operatorname{sinc}(s-$ $\left.\left.t_{j}\right)\right)_{j=-\infty}^{\infty}$ is a Riesz basis for its closed linear span. Then we say that $\left(t_{j}\right)_{j=-\infty}^{\infty}$ is an interpolating sequence.

Seip has produced an example of a frame $\left(\operatorname{sinc}\left(s-s_{j}\right)\right)_{j=-\infty}^{\infty}$ for $P W(\pi)$ such that no subsequence gives a Riesz basis for $P W(\pi)$; see $[25,26]$. We write

$$
Z+\ell^{2}=\left\{\left(t_{j}\right)_{j=-\infty}^{\infty}:\left(t_{j}-j\right)_{j=-\infty}^{\infty} \in \ell^{2}\right\}
$$

which is a complete metric space for the norm $\ell^{2}$.

Lemma 3.3 For $t=\left(t_{k}\right)_{k=-\infty}^{\infty}$, let $U_{t}: L^{2}[-\pi, \pi] \rightarrow L^{2}[-\pi, \pi]$ be the linear operator $U_{t}$ : $e^{i k x} \mapsto e^{i t_{k} x}$ for all $k \in \mathbf{Z}$. Then $U_{t}-I \in H S$ for all $t \in Z+\ell^{2}$, and

$$
\left\|U_{t}-U_{s}\right\|_{H S} \leq \frac{2 \pi}{\sqrt{3}}\|t-s\|_{\ell^{2}} \quad\left(s, t \in Z+\ell^{2}\right) .
$$

Proof. Clearly $U_{t}$ is determined by extending linearly its operation on the complete orthonormal basis $\left(e^{i k x}\right)_{k=-\infty}^{\infty}$ of $L^{2}([-\pi, \pi] ; d x / 2 \pi)$. By elementary estimates we have

$$
\begin{aligned}
\left\|U_{t}\left(e^{i k x}\right)-e^{i k x}\right\|_{L^{2}}^{2} & =\int_{-\pi}^{\pi}\left|e^{i t_{k} x}-e^{i k x}\right|^{2} \frac{d x}{2 \pi} \\
& \leq \frac{4 \pi^{2}}{3}\left(t_{k}-k\right)^{2}
\end{aligned}
$$

so $\sum_{k=-\infty}^{\infty}\left\|U_{t}\left(e^{i k x}\right)-e^{i k x}\right\|_{L^{2}}^{2}$ converges; hence $U_{t}-I$ is Hilbert-Schmidt. Likewise we obtain $\left\|U_{t}\left(e^{i k x}\right)-U_{s}\left(e^{i k x}\right)\right\|^{2} \leq 4 \pi^{2}\left(t_{k}-s_{k}\right)^{2} / 3$, which is a summable sequence whenever $t-s \in \ell^{2}$; hence $t \mapsto U_{t}$ is Lipschitz.

Proposition 3.4 Suppose that $(2 \pi / \sqrt{3})\left\|\left(t_{k}-k\right)_{k=-\infty}^{\infty}\right\|_{\ell^{2}}<1$. Then $\left(\operatorname{sinc}\left(x-t_{j}\right)\right)_{j=-\infty}^{\infty}$ is a Riesz basis for $R P W(\pi)$, so there exist $A, B>0$ such that for all for all $g \in R P W(\pi)$ there exists a unique $\left(a_{j}\right)_{j=-\infty}^{\infty} \in \ell^{2}$ such that $g(x)=\sum_{j=-\infty}^{\infty} a_{j} \operatorname{sinc}\left(x-t_{j}\right)$ converges in $L^{2}$ and

$$
A \int_{-\infty}^{\infty}|g(t)|^{2} d t \leq \sum_{j=-\infty}^{\infty}\left|g\left(t_{j}\right)\right|^{2} \leq B \int_{-\infty}^{\infty}|g(t)|^{2} d t \quad(g \in P W(\pi))
$$


Proof. Let $F: \ell^{2} \rightarrow L^{2}[-\pi, \pi]$ be the usual Fourier map $\left(a_{j}\right)_{j=-\infty}^{\infty} \mapsto \sum_{j=-\infty}^{\infty} a_{j} e^{i j x}$, and the Fourier transform $\mathcal{F} f(x)=\int_{-\pi}^{\pi} e^{-i x u} f(u) d u /(2 \pi)$, so that $F$ and $\sqrt{2 \pi} \mathcal{F}$ are unitary operators. Then $\mathcal{F} U_{t}: e^{i k x} \mapsto \operatorname{sinc}\left(x-t_{k}\right)$, and extending this linearly, we deduce that $S_{t}^{\dagger}=\mathcal{F} U_{t} F$ is a bounded linear operator.

In particular, with $Z=(j)_{j=-\infty}^{\infty}$ we obtain Shannon's map $S_{Z}: P W(\pi) \rightarrow \ell^{2}: f \mapsto$ $(f(j))_{j=-\infty}^{\infty}$, which is unitary [26, p. 209]. By Lemma 3.3, we have

$$
\begin{aligned}
\left\|S_{t}-S_{Z}\right\|_{o p} & \leq\left\|S_{t}^{\dagger}-S_{Z}^{\dagger}\right\|_{H S} \\
& \leq \frac{2 \pi}{\sqrt{3}}\left(\sum_{j=-\infty}^{\infty}\left(t_{j}-j\right)^{2}\right)^{1 / 2}
\end{aligned}
$$

so that

$$
\left\|S_{t} f\right\|_{\ell^{2}} \geq\|f\|_{L^{2}}-(2 \pi / \sqrt{3})\left\|\left(t_{k}-k\right)_{k=-\infty}^{\infty}\right\|_{\ell^{2}}\|f\|_{L^{2}}
$$

and likewise

$$
\left\|S_{t}^{\dagger}\left(a_{j}\right)\right\|_{\ell^{2}} \geq\left\|\left(a_{j}\right)_{j=-\infty}^{\infty}\right\|_{\ell^{2}}-(2 \pi / \sqrt{3})\left\|\left(t_{k}-k\right)_{k=-\infty}^{\infty}\right\|_{\ell^{2}}\left\|\left(a_{j}\right)_{j=-\infty}^{\infty}\right\|_{\ell^{2}}
$$

so that for $A=1-(2 \pi / \sqrt{3})\left\|\left(t_{k}-k\right)\right\|_{\ell^{2}}>0$ both the linear operators $S_{t}$ and $S_{t}^{\dagger}$ are embeddings. Hence $\left(\operatorname{sinc}\left(x-t_{j}\right)\right)_{j=-\infty}^{\infty}$ is a Riesz basis for its closed linear span, which is all of $P W(\pi)$.

\section{Concentration of measure on the tied spectrum}

The points of the tied spectrum consist of those $\mu \in \mathbf{R}$ such that there exists a nontrivial solution of $-f^{\prime \prime}+q f=\mu f$ with the Dirichlet boundary conditions $f(0)=0=f(2 \pi)$. These interlace the periodic spectrum, so we can assume that $\mu_{j}$ belongs to the $j^{\text {th }}$ interval of instability $\left(\lambda_{2 j-1}, \lambda_{2 j}\right)$ for $j=1,2, \ldots$ For a typical $q \in\left(\Omega_{N}, \nu_{N}^{\beta}\right)$, as $j \rightarrow \infty$ the length $\lambda_{2 j}-\lambda_{2 j-1}$ tends to zero, but the Lipschitz bounds on $q \mapsto \mu_{j}$ becomes larger; so we need to balance these effects a specially formulated concentration theorem.

Let $\xi_{j}=2 \sqrt{\mu_{j}}-j$, which is possibly complex for the first few $j$ and assuredly real thereafter, and form $\xi=\left(\xi_{j}\right)_{j=1}^{\infty}$. Also let

$$
d_{j}=C_{1} N(N+1) / j, \quad \alpha_{j}=2^{-1} j^{-8} \exp \left(-C \beta^{5 / 2} N^{9 / 4}\right) \quad(j=1,2, \ldots)
$$

for $C$ as in Lemma 2.2 and $C_{1}>0$ to be chosen.

Theorem 4.1 Let $F: \ell^{2} \rightarrow \mathbf{R}$ be 1-Lipschitz. Then

$$
\nu_{N}^{\beta}\left\{q \in \Omega_{N}: F(\xi(q))-\int_{\Omega_{N}} F(\xi) d \nu_{N}^{\beta}>s\right\} \leq e^{-c_{F}^{*}(s)} \quad(s \geq 0),
$$

where $c_{F}^{*}:[0, \infty) \rightarrow[0, \infty]$ is a convex function such that, for all $n=1,2, \ldots$,

$$
c_{F}^{*}(s) \geq \begin{cases}4^{-1} \alpha_{n}\left(s-d_{n}\right)^{2}, & \text { for all } s \geq d_{n} ; \\ 4^{-1}\left(1+\alpha_{n} d_{n}^{2}\right)^{-1} \alpha_{n} s^{2}, & \text { for all } d_{n}+1 /\left(\alpha_{n} d_{n}\right) \geq s \geq 0 .\end{cases}
$$


Theorem 4.1 gives a Gaussian concentration inequality, except that the constants change for the various ranges of $s$. In transportation theory, it is natural to have cost functions with a different shape for short distances, as in [33] page 593. The proof of Theorem 4.1 is split into three results in the remainder of this section, and begins by considering finitely many $\mu_{j}(q)$, as functions of $q \in \Omega_{N}$. First we prove that each $\mu_{j}$ is a Lipschitz function of $q$.

Lemma 4.2 For all $N>0$ there exist $C(N), \kappa_{2}>0$ such that $\Phi: q \mapsto\left(\mu_{1}(q), \mu_{2}(q), \ldots, \mu_{n}(q)\right)$ is Lipschitz from $\left(\Omega_{N}, L^{2}\right)$ to $\left(\ell_{n}^{2},\|\cdot\|_{\ell^{2}}\right)$ with constant $L(n, N) \leq \kappa_{2} C(N) n^{4}$.

Proof. Let $H S$ be the space of Hilbert-Schmidt operators on $L^{2}[0,2 \pi]$ with the usual norm. For $\zeta>N$, the operator $\zeta I-d^{2} / d x^{2}+q$ with boundary conditions $f(0)=f(2 \pi)=0$ is invertible and has inverse $G(q)$, and $G(q)$ is given by an integral operator with bounded Greens function. For these boundary conditions, we compute the Greens function for $f^{\prime \prime}=\zeta f$ and obtain

$$
G_{\zeta}(x, y)= \begin{cases}\frac{\sinh x \sqrt{\zeta} \sinh (2 \pi-y) \sqrt{\zeta}}{\sqrt{\zeta} \sinh 2 \pi \sqrt{\zeta}} & \text { for } 0 \leq x \leq y \leq 2 \pi \\ \frac{\sinh (2 \pi-x) \sqrt{\zeta} \sinh y \sqrt{\zeta}}{\sqrt{\zeta} \sinh 2 \pi \sqrt{\zeta}} & \text { for } 0 \leq y \leq x \leq 2 \pi .\end{cases}
$$

Moreover, from the identity $G(q)-G(p)=G(q)(p-q) G(p)$ and bounds on the Greens function, we obtain a constant $C(N)$ such that

$$
\|G(q)-G(p)\|_{H S} \leq C(N)\|p-q\|_{L^{2}}
$$

Real Lipschitz functions operate on differences of self-adjoint operators in the HilbertSchmidt norm, so for all Lipschitz functions $\varphi: \mathbf{R} \rightarrow \mathbf{R}$ such that $|\varphi(x)-\varphi(y)| \leq L|x-y|$, we have

$$
\|\varphi(G(q))-\varphi(G(p))\|_{H S} \leq \kappa_{2} L\|G(q)-G(p)\|_{H S} .
$$

By a result of Lidskii [28], the map $\Lambda: H S \rightarrow \ell^{2}$ which associates to a self-adjoint positive operator $A$ the decreasing list of eigenvalues gives a Lipschitz function. In particular,

$$
\|\Lambda(\varphi(G(q)))-\Lambda(\varphi(G(p)))\|_{\ell^{2}} \leq\|\varphi(G(q))-\varphi(G(p))\|_{H S} .
$$

The required Lipschitz function is partially specified by

$$
\varphi(y)= \begin{cases}0, & \text { if } y \leq 1 / 2 n^{2} \\ (1 / y)-\zeta, & \text { if } 1 / n^{2} \leq y \leq 1 / \zeta \\ 0, & \text { if } y \geq 2 / \zeta\end{cases}
$$

with straight line segments added to complete the graph and make the function continuous. Note that $\varphi(1 /(y+\zeta))=y$ and $\left|\varphi^{\prime}\right| \leq n^{4}$ in the middle of the domain, so

$$
\Lambda(\varphi(G(q)))=\left(\mu_{n}(q), \mu_{n-1}(q), \ldots, \mu_{1}(q), 0, \ldots\right)
$$


By combining the Lipschitz maps in (4.5), (4.6) and (4.7), we obtain the stated result.

Definition (Free energy) Let $(X, \mu)$ be a probability space and $F$ a real random variable on $(X, \mu)$ such that $F$ has finite mean. Define the normalized free energy of $F$ as $c_{F}: \mathbf{R} \rightarrow$ $(-\infty, \infty]$, where

$$
c_{F}(t)=\log \left(\int_{X} e^{t F(\xi)} \mu(d \xi)\right)-t \int_{X} F(\xi) \mu(d \xi) \quad(t \in \mathbf{R}) .
$$

Then $c_{F}$ is convex and its Legendre-Fenchel transform is defined by

$$
c_{F}^{*}(s)=\sup \left\{s t-c_{F}(t): t ; c_{F}(t)<\infty\right\}
$$

see [32] page 23. Also, $c_{F}^{*}$ is known as the (normalized) rate function.

The next step is to prove a concentration inequality for Lipschitz functions of finitely many tied eigenvalues.

Lemma 4.3 Let $\alpha(N, \beta)=2^{-1} \exp \left(-C \beta^{5 / 2} N^{9 / 4}\right), \xi_{j}=2 \sqrt{\mu_{j}}-j$, and for a 1-Lipschitz function $\Psi_{n}:\left(\mathbf{C}^{n}, \ell^{2}\right) \rightarrow \mathbf{R}$, let $F_{n}(q)=\Psi_{n}\left(\xi_{1}(q), \ldots, \xi_{n}(q)\right)$ on $\left(\Omega, \nu_{N}^{\beta}\right)$. Then $F_{n}$ satisfies the concentration inequality

$$
c_{F_{n}}(t) \leq \frac{\kappa_{2}^{2} C(N)^{2} n^{8} t^{2}}{2 \alpha(N, \beta)} \quad(t \in \mathbf{R}) .
$$

Proof. By Lemma 4.2, $F_{n}: \Omega_{N} \rightarrow \mathbf{R}$ is Lipschitz with constant $\kappa_{2} C(N) n^{4}$. By Corollary 2 of [3], any Lipschitz function on $\left(\Omega_{N}, \nu_{N}^{\beta}\right)$ satisfies a Gaussian concentration of measure inequality as in (4.11). Here $\alpha(N, \beta)$ is the logarithmic Sobolev constant of [3], Theorem 1. See [33, Theorem 22.10] for a detailed discussion.

Let $\left(d_{j}\right)_{j=1}^{\infty}$ be a decreasing positive sequence with $d_{j} \rightarrow 0$ as $j \rightarrow \infty$ and let

$$
X=\left\{\left(\xi_{j}\right)_{j=-\infty}^{\infty} \in \ell^{2}(\mathbf{C}):\left|\xi_{j}\right| \leq d_{j} / 2 ; j=1,2, \ldots\right\}
$$

Proposition 4.4 Let $\mu$ be a Radon probability measure on $X$, and suppose that there exists $\alpha_{n}>0$ such that $c_{F_{n}}(t) \leq t^{2} /\left(2 \alpha_{n}\right)$ for all 1-Lipschitz functions $F_{n}:\left(X, \ell^{2}\right) \rightarrow \mathbf{R}$ that depend only on the first $n$ coordinates. Then for all 1-Lipschitz functions $F:\left(X, \ell^{2}\right) \rightarrow \mathbf{R}$, the normalized free energy satisfies

$$
c_{F}(t) \leq \frac{t^{2}}{\alpha_{n}}+\frac{1}{2} \log \cosh \left(2 d_{n} t\right) \quad(t \in \mathbf{R})
$$

and the Legendre transform satisfies (4.2)

$$
c_{F}^{*}(s) \geq \begin{cases}4^{-1} \alpha_{n}\left(s-d_{n}\right)^{2} & \text { for all } s \geq d_{n} \\ 4^{-1}\left(1+\alpha_{n} d_{n}^{2}\right)^{-1} \alpha_{n} s^{2} & \text { for all } d_{n}+1 /\left(\alpha_{n} d_{n}\right) \geq s \geq 0\end{cases}
$$


Proof. Let $\mathcal{F}_{n}$ be the $\sigma$-algebra of Borel sets that is generated by the first $n$ coordinate functions on $X$, and for $F$ as above let $F_{n}=E\left(F \mid \mathcal{F}_{n}\right)$ be the conditional expectation with respect to $\mathcal{F}_{n}$ in $L^{2}(\mu)$; note that $F_{n}$ is 1 -Lipschitz and $\int F_{n} d \mu=\int F d \mu$. Now let

$$
Z_{n}(t)=\int_{X} e^{t\left(F-F_{n}\right)} d \mu
$$

which satisfies $Z_{n}(0)=1, Z_{n}^{\prime}(0)=0$ and $Z_{n}^{\prime \prime}(t)=\int_{X}\left(F-F_{n}\right)^{2} e^{t\left(F-F_{n}\right)} d \mu$, so $0 \leq Z_{n}^{\prime \prime}(t) \leq$ $d_{n}^{2} Z_{n}(t)$ and so $Z_{n}^{\prime}(t) \geq 0$ for all $t>0$. Integrating this differential inequality, we obtain $Z_{n}(t) \leq \cosh \left(d_{n} t\right)$. Then by the Cauchy-Schwarz inequality, we have

$$
\int_{X} e^{t\left(F-\int F d \mu\right)} d \mu \leq\left(\int_{X} e^{2 t\left(F-F_{n}\right)} d \mu\right)^{1 / 2}\left(\int_{X} e^{t\left(F_{n}-\int F_{n} d \mu\right)} d \mu\right)^{1 / 2}
$$

so that

$$
\begin{aligned}
c_{F}(t) & \leq(1 / 2) \log Z_{n}(2 t)+(1 / 2) c_{F_{n}}(2 t) \\
& \leq(1 / 2) \log \cosh \left(2 d_{n} t\right)+\alpha_{n}^{-1} t^{2} .
\end{aligned}
$$

To estimate $c_{F}^{*}(s)$, we first suppose that $0 \leq s \leq d_{n}+1 / \alpha_{n} d_{n}$, and use the estimate

$$
\begin{aligned}
c_{F}^{*}(s) & \geq \sup \left\{s t-c(t): 0<t<1 / 2 d_{n}\right\} \\
& \geq \sup \left\{s t-\alpha_{n}^{-1} t^{2}-d_{n}^{2} t^{2}: 0<t<1 / 2 d_{n}\right\} \\
& =\frac{\alpha_{n} s^{2}}{4\left(1+\alpha_{n} d_{n}^{2}\right)} .
\end{aligned}
$$

For $s \geq d_{n}$, we have

$$
c_{F}^{*}(s) \geq \sup \left\{s t-\alpha_{n}^{-1} t^{2}-d_{n} t: t>0\right\}=4^{-1} \alpha_{n}\left(s-d_{n}\right)^{2} .
$$

Proof of Theorem 4.1 As in (2.14), let $q \in \Omega_{N}$ be such that for some $C_{1}, C_{2}>0$ the periodic spectrum satisfies $\lambda_{2 j}-\lambda_{2 j-1} \leq C_{1} N|\hat{q}(j)|$ and $C_{2} j^{2} \leq \lambda_{2 j-1}$ for all $j=1,2, \ldots$ Also

$$
\sum_{j=n}^{\infty}\left|\xi_{j}\right|^{2}=\sum_{j=n}^{\infty}\left(\frac{4 \mu_{j}-j^{2}}{2 \sqrt{\mu_{j}}+j}\right)^{2} \leq \frac{(C N(N+1))^{2}}{C_{2} n}
$$

Hence $\xi \in X$ for the above $X$. We can apply Proposition 4.4 to the measure $\mu$ that is induced on $X$ from $\left(\Omega_{N}, \nu_{N}^{\beta}\right)$ by the map $q \mapsto \xi(q)$, since Lemma 4.3 implies that the hypotheses of Proposition 4.4 hold with the constants as in (4.1). We can therefore introduce $c_{F}^{*}$ as the Legendre-Fenchel transform of $c_{F}$, where $c_{F}$ is continuously differentiable on $\mathbf{R}$, and hence 
$\left\{s: c_{F}^{*}(s)<\infty\right\}$ contains the range of $c_{F}^{\prime}$. Theorem 4.1 follows from Proposition 4.4 by Chebyshev's inequality, since

$$
\nu_{N}^{\beta}\left\{q \in \Omega_{N}: F(\xi(q))-\int F(\xi) d \nu_{N}^{\beta}>s\right\} \leq e^{-s t+c_{F}(t)} \quad(s, t>0),
$$

and we can optimize this inequality over $t>0$ for each fixed $n$, and use the bounds (4.14).

\section{Transportation of measure and linear statistics}

Let $\tau=\left(\tau_{j}\right)_{j=-\infty}^{\infty}$ where

$$
\tau_{j}= \begin{cases}\sqrt{2\left(\lambda_{2 j}+\lambda_{2 j-1}\right)} & \text { for } j=1,2, \ldots \\ 0, & \text { for } j=0 ; \\ -\sqrt{2\left(\lambda_{-2 j}+\lambda_{-2 j-1}\right)} & \text { for } j=-1,-2, \ldots,\end{cases}
$$

and for $g \in R P W(\pi)$ introduce the linear statistic

$$
F(\tau)=\sum_{j=-\infty}^{\infty}\left(g\left(\tau_{j}\right)-g(j)\right)
$$

In this section we obtain bounds on $F(\tau)$ and its fluctuations. By Propositions 2.3 and 3.1, $\tau_{j}$ is close to $j$, and the new idea is that the fluctuations of $\sum_{j=-m}^{m}\left(g\left(\tau_{j}\right)-g(j)\right)$ are controlled, or at least attenuated, by $\sum_{j=-m}^{m}\left|g^{\prime}\left(\tau_{j}\right)\right|^{2}$. To make this precise, we introduce the infimum convolution, which generalizes the Legendre-Fenchel transform, and then we consider the special case of linear statistics.

Definition (Infimum convolution) For a continuous and bounded $F: \ell^{2} \rightarrow \mathbf{R}$, the Hopf-Lax infimum convolution is defined as in [5] by

$$
Q_{s} F(\eta)=\inf \left\{F(\xi)+\frac{1}{2 s}\|\xi-\eta\|^{2}: \xi \in \ell^{2}\right\} \quad\left(\eta \in \ell^{2}, s>0\right) .
$$

The purpose of the time parameter $s$ in $Q_{s}$ is to produce a semigroup that solves the Hamilton-Jacobi equation

$$
\frac{\partial}{\partial s} Q_{s} F=-(1 / 2)\left\|\nabla Q_{s} F\right\|^{2}
$$

Proposition 5.1 For $g \in R P W(\pi)$ with $\|g\|_{L^{2}} \leq 1$ and for $\xi=\left(\xi_{j}\right)_{j=-\infty}^{\infty} \in \ell^{2}$ let

$$
F(\xi)=\sum_{j=-\infty}^{\infty}\left(g\left(\xi_{j}+j\right)-g(j)\right)
$$

(i) Then $F$ is bounded, $F \mapsto Q_{s} F(\eta)$ is concave, and $Q_{s} F(\eta) \leq F(\eta)$ for all $s>0$ and $\eta \in \ell^{2}$;

(ii) the semigroup law $Q_{s+t} F=Q_{t} Q_{s} F$ holds for all $s, t>0$; 
(iii) $Q_{s} F(\eta) \rightarrow F(\eta)$ as $s \rightarrow 0+$; and

$$
\left(\frac{\partial}{\partial s}\right)_{s=0} Q_{s} F(\eta)=\frac{-1}{2} \sum_{j=-\infty}^{\infty} g^{\prime}\left(\eta_{j}+j\right)^{2} \quad\left(\eta=\left(\eta_{j}\right)_{j=-\infty}^{\infty} \in \ell^{2}\right) .
$$

Proof. (i) First we check that the series defining $F(\xi)$ is convergent and $F$ is bounded. By Fourier analysis, $g^{\prime} \in R P W(\pi)$. Also, by Proposition 3.4, for all $\xi=\left(\xi_{j}\right)_{j=-\infty}^{\infty} \in \ell^{2}$, we can perturb $(j)_{j=-\infty}^{\infty}$ to obtain a sampling sequence $\left(\xi_{j}+j\right)_{j=-\infty}^{\infty}$ for $R P W(\pi)$ by [25]. Hence there exists $B>0$ such that

$$
\sum_{j=-\infty}^{\infty}\left|g^{\prime}\left(j+\xi_{j}\right)\right|^{2} \leq B \int_{-\infty}^{\infty}\left|g^{\prime}(x)\right|^{2} d x
$$

for all $g \in R P W(\pi)$ and all $\xi \in \ell^{2}$ such that $\|\xi\|_{\ell^{2}} \leq 1$. From the Cauchy-Schwarz inequality, we deduce that $\sum_{j=1}^{\infty} g^{\prime}\left(j+\theta \xi_{j}\right) \xi_{j}$ converges absolutely for all $0<\theta<1$, and hence $\left.\sum_{j=-\infty}^{\infty} g\left(\xi_{j}+j\right)-g(j)\right)$ is absolutely convergent by the mean value theorem. The remaining statements are now straightforward.

(ii) This is a general property of the quadratic Hamilton-Jacobi semigroup [33, p. 584].

(iii) and (iv) We prove that for $0<s<\sqrt{5} / \pi^{2}$ there exists for each $\eta \in \ell^{2}$ a unique $\xi \in \ell^{2}$ in a neighbourhood of $\eta$ such that $Q_{s} F(\eta)=F\left(\left(\xi_{j}+j\right)_{j=-\infty}^{\infty}\right)+\left\|\left(\eta_{j}-\xi_{j}\right)_{j=-\infty}^{\infty}\right\|^{2} /(2 s)$. Using the Fourier representation, one can easily show that $g$ has bounded derivatives of order $k$ such that $\left\|g^{(k)}\right\|_{\infty} \leq \pi^{k}\|g\|_{L^{2}} / \sqrt{2 k+1}$ for $k=0,1, \ldots$ So for all $\eta_{j} \in \mathbf{R}, \sqrt{5} / \pi^{2}>s>0$ and $j \in \mathbf{Z}$, the real function $\xi_{j} \mapsto g\left(j+\xi_{j}\right)+\left(\xi_{j}-\eta_{j}\right)^{2} /(2 s)$ is differentiable and diverges to infinity as $\xi_{j} \rightarrow \pm \infty$, hence attains its infimum at a unique $\xi_{j}$ such that $s g^{\prime}\left(\xi_{j}+j\right)+\xi_{j}=\eta_{j}$ and $\left|\eta_{j}-\xi_{j}\right| \leq s \pi\|g\|_{L^{2}} / \sqrt{3}$. By the mean value theorem, we deduce that there exists $\zeta_{j}$ between $\xi_{j}$ and $\eta_{j}$ such that

$$
g\left(\xi_{j}+j\right)-g(j)+\frac{1}{2 s}\left(\xi_{j}-\eta_{j}\right)^{2}=g\left(\eta_{j}+j\right)-g(j)-\frac{s}{2} g^{\prime}\left(\xi_{j}+j\right)^{2}-\frac{1}{2} g^{\prime \prime}\left(\zeta_{j}+j\right)\left(\xi_{j}-\eta_{j}\right)^{2} .
$$

The map $T: \ell^{2} \rightarrow \ell^{2}$ given by $T:\left(\xi_{j}\right)_{j=-\infty}^{\infty} \mapsto\left(\xi_{j}+s g^{\prime}\left(\xi_{j}+j\right)\right)_{j=-\infty}^{\infty}$ is continuous and has Fréchet derivative $\nabla T(\xi)=\operatorname{diagonal}\left(1+s g^{\prime \prime}\left(\xi_{j}+j\right)\right)_{j=-\infty}^{\infty} ;$ so for $s \pi^{2}\|g\|_{L^{2}} / \sqrt{5}<1$, the operator $\nabla T$ is invertible on $\ell^{2}$, and hence $T$ is an open mapping. By summing (5.5) over $j$, we deduce that

$$
Q_{s} F(\eta)=F(\eta)-\frac{s}{2} \sum_{j=-\infty}^{\infty} g^{\prime}\left(\xi_{j}+j\right)^{2}+O\left(s^{2}\right),
$$

where the series converges to $\sum_{j=-\infty}^{\infty} g^{\prime}\left(j+\eta_{j}\right)^{2}$ as $s \rightarrow 0+$.

Theorem 5.2 There exists $\rho=\rho(N, \beta, m)>0$ such that for all $g \in R P W(\pi)$ with $\|g\|_{L^{2}} \leq 1$, the linear statistic $F_{m}(\tau)=\sum_{j=-m}^{m}\left(g\left(\tau_{j}\right)-g(j)\right)$ satisfies

$$
\int_{\Omega_{N}} \exp \left(\rho Q_{1} F_{m}(\tau(q))\right) \nu_{N}^{\beta}(d q) \leq \exp \left(\rho \int_{\Omega_{N}} F_{m}(\tau(q)) \nu_{N}^{\beta}(d q)\right) .
$$


Proof. By Proposition 2.3(ii), we can assume that all the periodic eigenvalues are simple. Then we note that $\lambda_{j}>0$ for all but finitely many indices $j$; whereas, in the exceptional cases where $\lambda_{2 j}<0$, we have $\tau_{j}$ purely imaginary so the sum $g\left(\tau_{j}\right)+g\left(\tau_{-j}\right)$ unambiguously gives a real random variable for all $g \in R P W(\pi)$.

We show that the solution operator of Hill's equation and hence the characteristic function are Lipschitz functions of $q \in \Omega_{n}$, so we can apply known concentration theorems to control some linear statistics. Let $p$ be an integer such that $m^{2}<p<(m+1)^{2}$, and let $S$ be the oriented square with vertices $\pm p \pm i p$ which is described once in the positive sense. Taking $m$ to be large, we can use the nonrandom bound $\|q\|_{L^{2}}^{2} \leq N$ to bound the terms in the series (3.7) uniformly for all $q \in \Omega_{N}$. We write Hill's equation in the style

$$
\left(\left[\begin{array}{cc}
0 & 1 \\
-1 & 0
\end{array}\right] \frac{d}{d x}-\frac{1}{2}\left[\begin{array}{cc}
1+q & -i(1-q) \\
-i(1-q) & -(1+q)
\end{array}\right]-\frac{\lambda}{2}\left[\begin{array}{ll}
1 & 0 \\
0 & 1
\end{array}\right]\right) \Psi_{\lambda}(x)=0
$$

Let

$$
R=\frac{1}{2}\left[\begin{array}{cc}
1+q & i q-i \\
i q-i & -1-q
\end{array}\right]
$$

The integral equation

$$
\Psi_{\lambda}(x)=\exp \left(x \sqrt{\lambda}\left[\begin{array}{cc}
0 & -1 \\
1 & 0
\end{array}\right]\right) \Psi_{\lambda}(0)+\int_{0}^{x} \exp \left((x-s) \sqrt{\lambda}\left[\begin{array}{cc}
0 & -1 \\
1 & 0
\end{array}\right]\right) R(s) \Psi_{\lambda}(s) d s
$$

has variational equation

$$
\begin{aligned}
\delta \Psi(x)= & \int_{0}^{x} \exp \left((x-s) \sqrt{\lambda}\left[\begin{array}{cc}
0 & -1 \\
1 & 0
\end{array}\right]\right) \delta R(s) \Psi_{\lambda}(s) d s \\
& +\int_{0}^{x} \exp \left((x-s) \sqrt{\lambda}\left[\begin{array}{cc}
0 & -1 \\
1 & 0
\end{array}\right]\right) R(s) \delta \Psi(s) d s .
\end{aligned}
$$

Then $\left\|\Psi_{\lambda}\right\| \leq M$. We choose $\kappa>0$ such that $\sqrt{\kappa N} e^{p \kappa}<1 / 2$ and split $[0,2 \pi]$ into consecutive subintervals of length $\kappa$; then we consider the supremum norm $\|\cdot\|_{\infty}$ on the matrix functions to obtain the bound

$$
\|\delta \Psi\|_{\infty} \leq \sqrt{\kappa}\left(\int_{0}^{\kappa}\|R(s)\|^{2} d s\right)^{1 / 2} e^{p \kappa}\|\delta \Psi\|_{\infty}+e^{p \kappa} \sqrt{\eta}\left(\int_{0}^{\kappa}\|\delta R(s)\|^{2} d s\right)^{1 / 2}\left\|\Psi_{\lambda}\right\|_{\infty}
$$

so that

$$
\|\delta \Psi\|_{\infty} \leq \frac{2}{\sqrt{N}}\left(\int_{0}^{\kappa}\|\delta R(s)\|^{2} d s\right)^{1 / 2}\|\Psi\|_{\infty} .
$$

We repeat this bound for each successive interval and thus we obtain a Lipschitz constant

$$
L_{\kappa} \leq\left(1+\frac{M}{2 \sqrt{N}}\right)^{2 \pi / \kappa}
$$


We deduce that $q \mapsto \Delta(\lambda)$ is Lipschitz continuous from $\left(\Omega_{N}, L^{2}\right)$ to the space of holomorphic functions inside $S$ with the uniform norm. The function $\lambda \mapsto \Psi_{\lambda}(s)$ is holomorphic and hence we can differentiate the integral equations (5.9) with respect to $\lambda$. By Cauchy's estimates, $q \mapsto \Delta^{\prime}(\lambda)$ is Lipschitz continuous.

We observe that there exists $\delta>0$ such that $\left|\Delta(\lambda)^{2}-4\right|>\delta$ for all $\lambda$ on $S$. Note that the zeros of $\Delta(\lambda)^{2}-4$ all lie on the real axis, and their position are described in the proof of Proposition 3.1. So we can form the product

$$
\Delta(\lambda)^{2}-4=c \prod_{j=0}^{\infty}\left(1-\frac{\lambda}{\lambda_{j}}\right)
$$

By estimating each factor on the compact set $S$, we obtain a lower bound $\delta>0$.

It follows from these estimates that the functions

$$
q \mapsto \frac{1}{\pi i} \int_{S} \frac{\Delta(\lambda) \Delta^{\prime}(\lambda) \lambda^{k} d \lambda}{\Delta(\lambda)^{2}-4} \quad(k=1, \ldots, m)
$$

are Lipschitz continuous on $\Omega_{N}$.

As in [4], we introduce the circles $C\left(n^{2} / 4,1 / 4\right)$ and apply Cauchy's integral formula to obtain

$$
\frac{\lambda_{2 j}+\lambda_{2 j-1}}{2}=\frac{1}{2 \pi i} \int_{C\left(n^{2} / 4,1\right)} \frac{\lambda \Delta(\lambda) \Delta^{\prime}(\lambda) d \lambda}{\Delta(\lambda)^{2}-4}
$$

for the midpoint of the $j^{\text {th }}$ interval of instability. Hence $q \mapsto\left(\lambda_{2 j}+\lambda_{2 j-1}\right) / 2$ is Lipschitz from $\left(\Omega_{N}, L^{2}\right) \rightarrow \mathbf{R}$ with constant $L_{j} \leq \kappa_{0}(N, \beta) j^{2}$ for some $\kappa_{0}(N, \beta)$ and all $j$. We deduce that $q \mapsto \tau_{j}$ is also Lipschitz. (It follows likewise that the functions $q \mapsto \sum_{j=1}^{2 m} \lambda_{j}^{k}$ are Lipschitz continuous for $k=1, \ldots$, but we have not quite proved that $q \mapsto \lambda_{j}^{k}$ is Lipschitz.)

Let $\mu_{m}$ be the probability measure that is induced from $\nu_{N}^{\beta}$ by the Lipschitz map $\varphi: q \mapsto$ $\left(\tau_{j}\right)_{j=-m}^{m}$, where the Lipschitz constant $L_{m}$ is finite. The measure $\nu_{N}^{\beta}$ satisfies a logarithmic Sobolev inequality

$$
\left.L S I(1 / \kappa): \quad \int_{\Omega_{N}} f(q)^{2} \log (f(q))^{2} / \int f^{2} d \nu_{N}^{\beta}\right) \nu_{N}^{\beta}(d q) \leq 2 \kappa \int_{\Omega_{N}}\|\nabla f(q)\|^{2} \nu_{N}^{\beta}(d q)
$$

by Theorem 1 of [3]. Now $\varphi$ induces $\mu_{m}$ on $\mathbf{R}^{2 m+1}$ from $\nu_{N}^{\beta}$ on $\Omega_{N}$. Also, by the chain rule applied to $f(q)=F_{m}(\varphi(q))$ we have $\|\nabla f(q)\| \leq L_{m}\left\|\left(\nabla F_{m}\right) \circ \varphi\right\|$, and hence we have the logarithmic Sobolev inequality such that

$$
\int_{\mathbf{R}^{2 m+1}} F_{m}(\xi)^{2} \log \left(F_{m}(\xi)^{2} / \int F_{m}^{2} d \mu_{m}\right) \mu_{m}(d \xi) \leq 2 L_{m}^{2} \kappa \int_{\mathbf{R}^{2 m+1}}\left\|\nabla F_{m}(\xi)\right\|^{2} \mu_{m}(d \xi)
$$

for all $C^{\infty}$ functions $F_{m}: \mathbf{R}^{2 m+1} \rightarrow \mathbf{R}$. In particular, this applies to the linear statistic $F_{m}$ of (5.7), since $\xi \mapsto Q_{t} F_{m}(\xi)$ is Lipschitz continuous and $t \mapsto Q_{t} F_{m}(\xi)$ is differentiable for almost 
all $\xi$, by [5, p 673]. The logarithmic Sobolev inequality (5.21) implies the transportation inequality (5.9) for $\mu_{m}$ with constant $\rho>0$ depending upon $L_{m}^{2} \kappa$ by [32, p. 292]. The crucial point is that

$$
\phi(t)=\frac{L_{m}^{2} \kappa}{t} \log \left(\int_{\mathbf{R}^{2 m+1}} e^{t Q_{t}\left(F_{m}\right)(\xi) / \kappa L_{m}^{2}} \mu_{m}(d \xi)\right)
$$

is a decreasing function of $0<t<1$ by the Hamilton-Jacobi equation and (5.21), so $\phi(1) \leq$ $\lim _{t \rightarrow 0+} \phi(t)$.

Corollary 5.3 There exists $\kappa(m, \beta, N)>0$ such that for all $g \in R P W(\pi)$ with $\|g\|_{L^{2}} \leq 1$, the linear statistic $F_{m}(\tau)=\sum_{j=-m}^{m}\left(g\left(\tau_{j}\right)-g(j)\right)$ satisfies

$$
\nu_{N}^{\beta}\left\{q \in \Omega_{N}: F_{m}(\tau(q))-\int F_{m} d \nu_{N}^{\beta}>\varepsilon\right\} \leq e^{-\kappa(m, \beta, N) \varepsilon^{2}} \quad(\varepsilon>0) .
$$

Proof. This follows from Theorem 5.2 by [33, Theorem 22.22].

Proposition 5.4 For $1>\beta>0$ and $N_{1}>N>0$, there exist $\kappa_{1}(N, \beta)>0$ and $\kappa_{2}(N, \beta)>0$ such that, for all $g \in R P W(\pi)$ with $\|g\|_{L^{2}} \leq 1$, the corresponding linear statistic satisfies

$$
\nu_{N}^{\beta}\left\{q \in \Omega_{N}: F(\tau(q))-\int F d \nu_{N}^{\beta}>\varepsilon\right\} \leq \exp \left(-\kappa_{1}(n, \beta) \varepsilon^{2} \min \left\{1, \kappa_{2}(N, \beta) \varepsilon^{4}\right\}\right) \quad(\varepsilon>0) .
$$

Proof. Let $F_{m}(\tau)$ be the partial sum $F_{m}(\tau)=\sum_{j=-m}^{m}\left(g\left(\tau_{j}\right)-g(j)\right)$. Now we use the mean value theorem and the Cauchy-Schwarz inequality to bound

$$
\begin{aligned}
\left|F(\tau)-F_{m}(\tau)\right| & =\left|\sum_{j:|j| \geq m+1}\left(g\left(\tau_{j}\right)-g(j)\right)\right| \\
& \leq\left(\sum_{j:|j| \geq m+1}\left|\tau_{j}-j\right|^{2}\right)^{1 / 2}\left(\sum_{j:|j| \geq m+1}\left|g^{\prime}\left(\eta_{j}\right)\right|^{2}\right)^{1 / 2} \\
& \leq\left(\sum_{j:|j| \geq m+1}\left|\frac{\tau_{j}^{2}-j^{2}}{j+\tau_{j}}\right|^{2}\right)^{1 / 2}\left(\sum_{j=-\infty}^{\infty}\left|g^{\prime}\left(\eta_{j}\right)\right|^{2}\right)^{1 / 2},
\end{aligned}
$$

for some $\eta_{j}$ between $j$ and $\tau_{j}$. Then we use (5.4) and (3.1) to obtain the bound

$$
\left|F(\tau)-F_{m}(\tau)\right| \leq m^{-1} B(C(\beta) N(N+1))^{1 / 2}\left\|g^{\prime}\right\|_{L^{2}}
$$

for some constant $C(\beta)$, and $\left\|g^{\prime}\right\|_{L^{2}} \leq \pi\|g\|_{L^{2}}$.

Hence, in the decomposition

$$
\left[F-\int F>\varepsilon\right] \subseteq\left[F_{m}-\int F_{m}>\varepsilon / 3\right] \cup\left[\int F_{m}-\int F>\varepsilon / 3\right] \cup\left[F-\int F_{m}>\varepsilon / 3\right]
$$

we can ensure that the final two events are empty by selecting $m \geq \max \{1, \kappa / \varepsilon\}$. 
For such an $m$, the function $q \mapsto F_{m}(\tau)$ is Lipschitz with constant of order $m^{2}$, so by Corollary 2 of [3], there exists $\alpha(N, \beta)$ such that

$$
\nu_{N}^{\beta}\left\{q \in \Omega_{N}: F_{m}-\int F_{m} d \nu_{N}^{\beta}>\eta\right\} \leq \exp \left(-\alpha(N, \beta) \eta^{2} / m^{4}\right) \quad(\eta>0) .
$$

We choose $\eta=\varepsilon / 3$ to conclude the proof.

\section{Riesz bases}

The purpose of this section is to prove the following theorem concerning sampling from PaleyWiener space on a sequence given by the random eigenvalues, as in (1.9).

Definition (Gram matrix) For a real sequence $t=\left(t_{j}\right)_{j=-\infty}^{\infty}$, the Gram matrix of the sequence $\left(\operatorname{sinc}\left(x-t_{j}\right)\right)_{j=-\infty}^{\infty}$ in $R P W(\pi)$ is

$$
\Gamma(t)=\left[\operatorname{sinc}\left(t_{j}-t_{k}\right)\right]_{j, k=-\infty}^{\infty}
$$

Theorem 6.1 Let $t=\left(t_{j}\right)_{j=-\infty}^{\infty}$ be the modified eigenvalues of Hill's equation as in (1.9). There exist $\beta>0$ and $N>0$ such that on a set of strictly positive $\nu_{N}^{\beta}$ measure,

(i) $\left(\operatorname{sinc}\left(t-t_{j}\right)\right)_{j=-\infty}^{\infty}$ gives a Riesz basis for $R P W(\pi)$;

(ii) there exists a corresponding system of biorthogonal functions $\left(g_{j}\right)_{j=-\infty}^{\infty}$ such that for all $f \in R P W(\pi)$, the series

$$
f(x)=\sum_{j=-\infty}^{\infty}\left\langle f, g_{j}\right\rangle \operatorname{sinc}\left(x-t_{j}\right)
$$

converges in $L^{2}$, and $\left(\left\langle f, g_{j}\right\rangle_{L^{2}}\right)_{j=-\infty}^{\infty} \in \ell^{2}$;

(iii) the Gram matrix $\Gamma(t)$ is invertible, and belongs to $I+H S$;

(iv) the Carleman determinant satisfies

$$
\operatorname{det}_{2} \Gamma(t)=\lim _{n \rightarrow \infty}\left|\operatorname{det}\left[\operatorname{sinc}\left(t_{j}-k\right)\right]_{j, k=-n}^{n}\right|^{2}>0
$$

The main idea of this section is to analyse $\Gamma(t)$ in the space $I+H S$, about which we record some facts. First, $I+H S$ is a complete metric space for the $H S$ norm. By elementary functional calculus, one can carry out a type of polar decomposition in $I+H S$. The group $\mathcal{G}=\left\{X \in I+H S: \operatorname{det}_{2} X \neq 0\right\}$ contains a subgroup $\mathcal{K}=\left\{W \in \mathcal{G}: W^{\dagger} W=W^{\dagger}=I\right\}$ and a convex set $\mathcal{P}_{+}=\left\{G \in \mathcal{G}: G=G^{\dagger}, G \geq 0\right\}$. The unitary group acts on each of $\mathcal{G}, \mathcal{K}$ and $\mathcal{P}_{+}$by $(W, X) \mapsto W X W^{\dagger}$. In particular, for each $G \in \mathcal{P}_{+}$there exists a unitary $W$ and a diagonal operator $D=\operatorname{diag}\left(x_{j}\right) \in \mathcal{P}_{+}$with eigenvalues $x_{j}>0$ and $\sum_{j}\left(x_{j}-1\right)^{2}$ finite such that $G=W D W^{\dagger}$. Also $\mathcal{K} \times \mathcal{K}$ acts on $\mathcal{G}$ by $(U, V): X \mapsto U X V^{\dagger}$ for $U, V \in \mathcal{K}$ and $X \in \mathcal{G}$, and the map $\mathcal{K} \times \mathcal{K} \times \mathcal{P}_{+} \rightarrow \mathcal{G}:(U, V, G) \mapsto U G V^{\dagger}$ is surjective. The space $\mathcal{P}_{+}$is introduced to describe Gram matrices. 
Lemma 6.2 The Gram matrix $\Gamma(t)$ belongs to $I+H S$, and $\operatorname{det}_{2} \Gamma(t)$ is a Lipschitz function of $t$ on bounded subsets of $Z+\ell^{2}$.

Proof. In the notation of Lemma 3.3, $U_{t}-I$ and hence $U_{t}^{\dagger} U_{t}-I$ are Hilbert-Schmidt. Taking the inverse Fourier transform $\mathcal{F}: L^{2}[-\pi, \pi] \rightarrow R P W(\pi)$, we deduce that

$$
\Gamma(t)=\left[\left\langle U_{t}\left(e^{i j x}\right), U_{t}\left(e^{i k x}\right)\right\rangle\right]_{j, k=-\infty}^{\infty}
$$

belongs to $\left\{G \in I+H S: G=G^{\dagger}, G \geq 0\right\}$ for all $t \in Z+\ell^{2}$, and has a Carleman determinant.

By Lemma 3.3, $\left(t_{j}\right) \mapsto U_{t}$ is Lipschitz on $Z+\ell^{2}$, with $\left\|U_{t}-U_{s}\right\|_{H S} \leq 2 \pi\|t-s\|_{\ell^{2}} / \sqrt{3}$, so $\left(t_{j}\right) \mapsto U_{t}^{\dagger} U_{t}$ is Lipschitz on bounded subsets of $Z+\ell^{2}$. On the convex bounded set $\left\{A:\|A\|_{H S} \leq M\right\}$, the function $A \mapsto \operatorname{det}_{2}(I+A)$ is Lipschitz with constant $L \leq e^{c(2 M+1)^{2}}$, where $c>0$ is some universal constant. Hence $t \mapsto \operatorname{det}_{2} U_{t}^{\dagger} U_{t}$ is Lipschitz continuous on bounded sets.

Proof of Theorem 6.1 (i) and (iii). Then $\Gamma(t)$ represents $S_{t} S_{t}^{\dagger}$ with respect to the standard orthonormal basis of $\ell^{2}$. To prove that $S_{t}^{\dagger}$ is an embedding, it suffices to show that $\operatorname{det}_{2} \Gamma(t)>0$ on a set of positive measure. To do this, we choose $0<\delta<1 / 2$ and then $n>(\beta N)^{1 / \delta} \kappa^{1 / \delta}$ as in Proposition 3.1, and reduce the analysis to a finite rank operator $A_{n}$.

By Proposition 3.1, for all $N$, there exists $M$ such that $\left\|\left(t_{j}-j\right)\right\|_{\ell^{2}} \leq M$ for all $\left(t_{j}(q)\right)$ that arise as sampling sequences for $q \in \Omega_{N}$. By Lemma 6.2, there exists $L>0$ such that $t \mapsto \operatorname{det}_{2} \Gamma(t)$ is $L$-Lipschitz on $\left\{t=\left(t_{j}\right):\left\|\left(t_{j}-j\right)\right\|_{\ell^{2}} \leq M\right\}$.

We introduce the finite-rank operator $A_{n}: L^{2}[-\pi, \pi] \rightarrow L^{2}[-\pi, \pi]$ such that

$$
A_{n}\left(e^{i k x}\right)= \begin{cases}e^{i t_{k} x}-e^{i k x}, & \text { for } k=-n, \ldots, n \\ 0 & \text { else }\end{cases}
$$

and write $U_{t}$ from Lemma 6.1 as $U_{t}=I+A_{n}+B_{n}$. Observe that $q \mapsto A_{n}$ gives a Lipschitz map $\Omega_{N} \rightarrow H S$, such that $0 \mapsto 0$. Note also that $\left[\operatorname{sinc}\left(t_{j}-t_{k}\right)\right]_{j, k=-n}^{n}$ is a block submatrix of $\left(I+A_{n}^{\dagger}\right)\left(I+A_{n}\right)$, and that $\left\langle\left(I+A_{n}^{\dagger}\right)\left(I+A_{n}\right)\left(e^{i k x}\right), e^{i k x}\right\rangle=1$ for all $k \in \mathbf{Z}$, so

$$
\begin{aligned}
\operatorname{det}_{2}\left(I+A_{n}^{\dagger}\right)\left(I+A_{n}\right) & =\left(\operatorname{det}\left(I+A_{n}^{\dagger}\right)\left(I+A_{n}\right)\right) e^{-\operatorname{trace}\left(A_{n}+A_{n}^{\dagger}+A_{n}^{\dagger} A_{n}\right)} \\
& =\left|\operatorname{det}\left(I+A_{n}\right)\right|^{2} \\
& =\left|\operatorname{det}\left[\operatorname{sinc}\left(t_{j}-k\right)\right]_{j, k=-n}^{n}\right|^{2},
\end{aligned}
$$

a formula reminiscent of kernels from random matrix theory [14, p 124]. Then by Lemma 6.2,

$$
\begin{aligned}
\int_{\Omega_{N}} \operatorname{det} \Gamma(t) \nu_{N}^{\beta}(d q) \geq & \int_{\Omega_{N}} \operatorname{det}_{2}\left(\left(I+A_{n}^{\dagger}\right)\left(I+A_{n}\right)\right) \nu_{N}^{\beta}(d q) \\
& -L \int_{\Omega_{N}}\left(2(1+M)\left\|B_{n}\right\|_{H S}+\left\|B_{n}\right\|_{H S}^{2}\right) \nu_{N}^{\beta}(d q)
\end{aligned}
$$


where, as in (6.5),

$$
\left\|B_{n}\right\|_{H S} \leq \frac{2 \pi}{\sqrt{3}}\left(\sum_{k:|k| \geq n}\left(t_{k}-k\right)^{2}\right)^{1 / 2}
$$

and

$$
\left\|A_{n}\right\|_{H S} \leq \frac{2 \pi}{\sqrt{3}}\left(\sum_{k=-n}^{n}\left(t_{k}-k\right)^{2}\right)^{1 / 2} .
$$

By Proposition 3.1, we have $\sum_{k=n}^{\infty}\left(t_{k}-k\right)^{2} \leq C \beta N n^{2 \delta-1} /(1-2 \delta)$ so we can choose $n$ so large that the final integral in (7.14) is less than $1 / 8$. Then we choose $N, \beta>0$ so small that

$$
\begin{aligned}
\int_{\Omega_{N}} \operatorname{det}_{2}\left(\left(I+A_{n}^{\dagger}\right)\left(I+A_{n}\right)\right) \nu_{N}^{\beta}(d q) & \geq 1-\frac{2 L \pi}{\sqrt{3}} \int_{\Omega_{N}}\left(\sum_{k=-n}^{n}\left(t_{k}-k\right)^{2}\right)^{1 / 2} \nu_{N}^{\beta}(d q) \\
& >3 / 4
\end{aligned}
$$

hence (6.7) is greater than $1 / 8$ some some $N, \beta>0$. Consequently $\operatorname{det}_{2} \Gamma(t)>1 / 8$ on an open set that has strictly positive $\nu_{N}^{\beta}$ measure and there $\left(\operatorname{sinc}\left(s-t_{j}\right)\right)_{j=-\infty}^{\infty}$ gives a Riesz basis for its linear span. Since $\left\|B_{n}\right\| \rightarrow 0$ as $n \rightarrow \infty$, we can arrange for $U_{n}$ to be invertible, so the linear span is all of $R P W(\pi)$.

(iv) As $n \rightarrow \infty$, we have $\left\|B_{n}\right\|_{H S} \rightarrow 0$, and hence $\left|\operatorname{det}\left(I+A_{n}\right)\right|^{2} \rightarrow \operatorname{det}_{2} \Gamma(t)$.

(ii) There exists an invertible linear operator $W_{t}: L^{2}[-\pi, \pi] \rightarrow R P W(\pi)$ such that $W_{t}\left(e^{i j x}\right)=\operatorname{sinc}\left(s-t_{j}\right)$ and then $\left(g_{j}\right)_{j=-\infty}^{\infty}$ where $g_{j}=\left(W_{t}^{\dagger}\right)^{-1}\left(e^{i j x}\right)$ gives a system of biorthogonal functions for $\left(\operatorname{sinc}\left(s-t_{j}\right)\right)_{j=-\infty}^{\infty}$. Note that $\left(g_{j}\right)_{j=-\infty}^{\infty}$ is itself a Riesz basis, with $\left\|g_{j}\right\|_{L^{2}} \leq M_{1}$ for some $M_{1}$ and all $j \in \mathbf{Z}$, and $\left[\operatorname{sinc}\left(t_{j}-t_{k}\right)\right]$ has inverse matrix $\left[\left\langle g_{j}, g_{k}\right\rangle\right]$.

Remarks 6.3 (i) In [26] page 124, Katz and Sarnak considered a version of the Gram matrix $\Gamma(t)$ when the entries arise from eigenvalues of random unitary operators. The determinant of (6.3) resembles some expressions which appear in the representation theory of classical groups as in [14] page 122 , especially when written in the style

$$
\begin{gathered}
\operatorname{det}\left[\operatorname{sinc}\left(t_{j}-k\right)\right]_{j, k=-n}^{n} \\
=\frac{1}{(2 n+1) !} \int_{[-\pi, \pi]^{2 n+1}} \frac{\operatorname{det}\left[e^{i t_{j} x_{k}}\right]_{j, k=-n}^{n}}{\operatorname{det}\left[e^{i j x_{k}}\right]_{j, k=-n}^{n}} \prod_{-n \leq j<k \leq n}\left|e^{i x_{j}}-e^{i x_{k}}\right|^{2} \frac{d x_{-n}}{2 \pi} \ldots \frac{d x_{n}}{2 \pi} .
\end{gathered}
$$

This integral formula follows from Andréief's identity and the usual Vandermonde determinant.

(ii) In terms of Theorem $\mathrm{E}$ of [25], the phase function for the sampling sequence $\left(t_{j}\right)_{j=-\infty}^{\infty}$ is, for some real $\alpha$,

$$
\varphi(x)=\alpha+\operatorname{sign}(x) \int_{\left\{\lambda \in\left[0, x^{2}\right]: 4-\Delta(\lambda)^{2} \geq 0\right\}} \frac{\left|\Delta^{\prime}(\lambda)\right| d \lambda}{\sqrt{4-\Delta(\lambda)^{2}}}
$$


so that $\varphi$ is continuous, increasing, constant when $x^{2}$ belongs to an interval of instability, and increases by $\pi$ as $x^{2}$ increases over each interval of stability. These properties follow from the fact that $\Delta^{\prime}(\lambda)$ is of constant sign on each interval of stability by Laguerre's theorem.

\section{The Jacobian and linear statistics}

In this section we consider the set $\mathcal{M}_{\lambda}$ of potentials $q \in \Omega_{N}$ that have a given periodic spectrum $\lambda=\left(\lambda_{j}\right)_{j=0}^{\infty}$. Hochstadt [21, p. 219] observed that if only finitely many of the zeros of $\Delta(x)^{2}-4$ are simple, so that the spectrum of $q$ has only finitely many gaps, then $\Delta^{\prime}(x) / \sqrt{\Delta(x)^{2}-4}$ is an algebraic function. However, for typical $q$ in $\left(\Omega_{N}, \nu_{N}^{\beta}\right)$, Proposition 2.3(ii) shows that all the periodic eigenvalues are simple, so Hill's curve $\mathcal{E}$ of (1.11) is a hyperelliptic transcendental Riemann surface that has infinite genus. In Lemma 7.1 we define a suitable space of divisors on $\mathcal{E}$, and a map which associates to each $q \in \mathcal{M}_{\lambda}$ a divisor $\delta$ on $\mathcal{E}$ which is determined by the tied spectrum $\left(\mu_{j}\right)_{j=1}^{\infty}$ of $q$. Moreover, there is a pairing of divisors with the differential $\omega_{\infty}=\left(\Delta^{\prime}(x) / \sqrt{\Delta(x)^{2}-4}\right) d x$. The main task is to interpret (1.12) and the addition rule on divisors. Clearly, translating $q(x)$ to $q(x+s)$ preserves the periodic spectrum, and the measure $\nu_{N}^{\beta}$, but changes the tied spectrum. Suppose that $\lambda_{2}>0$, let $t_{j}=\sqrt{\lambda_{2 j}}$ for $j=1,2, \ldots, t_{0}=0$ and $t_{-j}=-t_{j}$ and suppose further that $\left(\operatorname{sinc}\left(s-t_{j}\right)\right)$ is a Riesz basis for $R P W(\pi)$. By Theorem 6.1, this event has positive probability with respect to $\nu_{N}^{\beta}$ for suitable $\beta, N>0$. Using the classical language of divisors, we analyse the addition rule on the expressions $\sum_{j=-\infty}^{\infty} x_{j} g\left(t_{j}\right)$.

The Jacobian of $\mathcal{E}$ is a complex torus of infinite dimension which has real part $\mathbf{X}$. To construct the map from the divisors on $\mathcal{E}$ to $\mathbf{X}$, McKean and Trubowitz [22] used sampling on a space of entire functions similar to $P W(\pi)$ and thus obtained a suitable family of holomorphic differentials. Their theory requires smooth $q$, so we extend this to typical $q$ in $\left(\Omega_{N}, \nu_{N}^{\beta}\right)$. In this section we define $\mathbf{X}$ in terms of sampling on $R P W(\pi)$. We also show that the Jacobian map has a Carleman determinant, with a rescaling argument to avoid the formal computations of section 12 of [22].

We momentarily suppose that $\mu_{j}=\lambda_{2 j}$ for all but finitely many $j$ and then in Theorem 7.3 allow more general assumptions. By analogy with classical examples [23], we introduce the generating function

$$
S=\sum_{j} 2 \int_{\mu_{j}}^{\lambda_{2 j}} \sqrt{\Delta(x)^{2}-4} d x
$$

and introduce the Taylor coefficients of $\Delta(x)^{2}-4=\sum_{j=0}^{\infty} \alpha_{j} x^{j}$ as new variables. The corresponding phases are defined by

$$
\varphi_{k}=\frac{\partial S}{\partial \alpha_{k}}=\sum_{j=1}^{\infty} \int_{\mu_{j}}^{\lambda_{2 j}} \frac{x^{k} d x}{\sqrt{\Delta(x)^{2}-4}} \quad(k=0,1, \ldots)
$$


Then we associate with an entire function $h(x)=\sum_{k=0}^{\infty} \beta_{k} x^{k}$ the series

$$
\sum_{k=0}^{\infty} \beta_{k} \varphi_{k}=\sum_{j=1}^{\infty} \int_{\mu_{j}}^{\lambda_{2 j}} \frac{h(x) d x}{\sqrt{\Delta(x)^{2}-4}}
$$

A real point $\mathbf{q}_{j}$ on $\mathcal{E}$ has the form $\mathbf{q}_{j}=\left(\mu_{j}, \varepsilon_{j} \sqrt{\Delta\left(\mu_{j}\right)^{2}-4}\right)$ where $\lambda_{2 j-1} \leq \mu_{j} \leq \lambda_{2 j}$ is in the $j^{\text {th }}$ spectral gap, $\sqrt{\Delta\left(\mu_{j}\right)^{2}-4} \geq 0$ and $\varepsilon_{j}= \pm 1$ where the signs indicate the top and bottom of the cut in $\mathcal{E}$. In particular, let $\mathbf{p}_{j}=\left(\lambda_{2 j}, 0\right)$, and, for any subset $M$ of $\mathbf{N}$, introduce the real divisor $\delta=\sum_{j \in M}\left(\mathbf{p}_{j}-\mathbf{q}_{j}\right)$. The set of such $\delta$ generates a free abelian group Div under formal addition and subtraction, which we regard as real divisors of degree zero. When

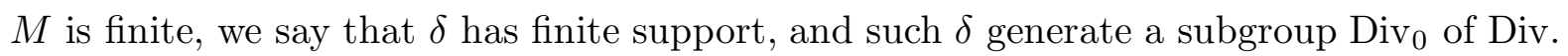

Lemma 7.1 Let $V=\left\{g \in R P W(\pi): z^{2} g(z) \in R P W(\pi)\right\}$ with $\|g\|_{V}^{2}=\|g\|_{L^{2}}^{2}+\left\|z^{2} g(z)\right\|_{L^{2}}^{2}$ have dual space $V^{*}$ with respect to the pairing $\langle f, g\rangle_{L^{2}}$.

(i) Then for all $\delta \in$ Div there exists $\psi_{\delta} \in V^{*}$, given by

$$
\psi_{\delta}(g)=\sum_{j \in M} \varepsilon_{j} \int_{\mu_{j}}^{\lambda_{2 j}} \frac{(g(\sqrt{x})+g(-\sqrt{x})) d x}{\sqrt{\Delta(x)^{2}-4}}
$$

such that $J: \delta \mapsto \psi_{\delta}$ is a group homomorphism Div $\rightarrow V^{*}$.

(ii) Let $g_{\varepsilon}(z)=2^{-1} \Delta^{\prime}\left((1-6 \varepsilon) z^{2}\right)(\operatorname{sinc}(\varepsilon z))^{3}$. Then $g_{\varepsilon} \in V$ for all $\varepsilon>0$ and the limit

$$
\psi_{\delta}\left(g_{\varepsilon}\right) \rightarrow \omega_{\infty}(\delta)=\sum_{j \in M} \varepsilon_{j} \int_{\mu_{j}}^{\lambda_{2 j}} \frac{\Delta^{\prime}(x) d x}{\sqrt{\Delta(x)^{2}-4}} \quad(\varepsilon \rightarrow 0+)
$$

exists for all $\delta \in \operatorname{Div}_{0}$, and defines a group homomorphism $\omega_{\infty}: \operatorname{Div}_{0} \rightarrow \mathbf{R}$.

(iii) For any sequence of signs $\varepsilon_{j}= \pm 1$, there is a map $\mathcal{M}_{\lambda} \rightarrow$ Div given by $q \mapsto \sum_{j=1}^{\infty}\left(\mathbf{p}_{j}-\mathbf{q}_{j}\right)$ where $\left(\mu_{j}\right)_{j=1}^{\infty}$ is the tied spectrum of (1.1) for $q$ and $\mathbf{q}_{j}=\left(\mu_{j}, \varepsilon_{j} \sqrt{\Delta\left(\mu_{j}\right)^{2}-4}\right)$.

Proof. (i) Note that $g(\sqrt{x})+g(-\sqrt{x})=(2 / \pi) \int_{0}^{\pi} \cos (s \sqrt{x}) \Re \hat{g}(s) d s$, so the numerator in (7.4) is entire of order $1 / 2$. Now Div gives a subgroup of $V^{*}$ since $\psi_{\delta}$ defines a bounded linear functional on $V$; indeed, we can bound the $j^{\text {th }}$ summand by a constant multiple of

$$
\int_{\mu_{j}}^{\lambda_{2 j}} \frac{|g(\sqrt{x})+g(-\sqrt{x})| d x}{\sqrt{\left(1-x / \lambda_{2 j}\right)\left(x / \lambda_{2 j-1}-1\right)}}
$$

where $\sum_{j=1}^{\infty} j^{2}\left|g\left(\sqrt{\lambda_{2 j}}\right)\right|$ converges by (3.25) and the Cauchy-Schwarz inequality. Thus there exists a uniquely determined sequence $\left(x_{k}(\delta)\right)_{k=-\infty}^{\infty}$ such that $\left(x_{k}(\delta) /\left(1+k^{2}\right)\right)_{k=-\infty}^{\infty} \in \ell^{2}$ and

$$
\sum_{k=-\infty}^{\infty} x_{k}(\delta) g\left(t_{k}\right)=\sum_{j=1}^{\infty} \varepsilon_{j} \int_{\mu_{j}}^{\lambda_{2 j}} \frac{(g(\sqrt{x})+g(-\sqrt{x})) d x}{\sqrt{\Delta(x)^{2}-4}} \quad(g \in V)
$$


so with respect to this basis $\psi_{\delta}=\sum_{j=-\infty}^{\infty} x_{j}(\delta) \operatorname{sinc}\left(s-t_{j}\right) \in V^{*}$. This correspondence respects the group law, so that if $\delta \mapsto\left(x_{j}(\delta)\right)$ and $\varepsilon \mapsto\left(y_{j}(\varepsilon)\right)$, then $\delta-\varepsilon \mapsto\left(x_{j}(\delta)-y_{j}(\varepsilon)\right)$. For $\delta \in \operatorname{Div}_{0}$, we can recover the coefficients by operating on the biorthogonal functions, obtaining $\psi_{\delta}\left(g_{j}\right)=x_{j}(\delta)$.

In particular, there exist bounded linear functionals

$$
\psi_{\left(\lambda_{2 j}, 0\right)-\left(\lambda_{2 j-1}, 0\right)}: g \mapsto 2 \int_{\lambda_{2 j-1}}^{\lambda_{2 j}} \frac{g(\sqrt{x})+g(-\sqrt{x})}{\sqrt{\Delta(x)^{2}-4}} d x \quad(g \in V)
$$

classically known as the real periods. By extension, $\sum_{j \in M} \psi_{\left(\lambda_{2 j}, 0\right)-\left(\lambda_{2 j-1}, 0\right)}$ is also a bounded linear functional on $V$ for all subsets $M$ of $\mathbf{N}$. Let $\Lambda$ be the lattice in $V^{*}$ that is generated by $\left\{\sum_{j \in M} \psi_{\left(\lambda_{2 j}, 0\right)-\left(\lambda_{2 j-1}, 0\right)}: M \subseteq \mathbf{N}\right\}$.

(ii) By Theorem 2.1 of [20], all of the zeros of $4-\Delta(z)^{2}$ are real, so by Laguerre's theorem, all the zeros of $\Delta^{\prime}(z)$ are also real, and separated by the zeros of $4-\Delta(z)^{2}$; see [10, p. 264]. Hence from the resulting product representation, $\Delta^{\prime}\left(z^{2}\right)$ is even and entire of exponential type; also $\Delta^{\prime}(z)$ is real for all real $z$. By (3.9) and the Cauchy integral formula, we deduce that $\Delta^{\prime}\left(z^{2}\right)$ is bounded on $\mathbf{R}$ as in [10, p 264], so $x^{2} g_{\varepsilon}(x), g_{\varepsilon}(x) \in L^{2}(\mathbf{R})$; hence $z g_{\varepsilon}(z), g_{\varepsilon}(z) \in R P W(\pi)$. Also, $g_{\varepsilon}(z) \rightarrow 2^{-1} \Delta^{\prime}\left(z^{2}\right)$ as $\varepsilon \rightarrow 0+$, uniformly on compact subsets of $\mathbf{C}$. We observe that, for all $\delta, \eta \in \operatorname{Div}_{0}$, we have $\omega_{\infty}(\delta+\eta)=\omega_{\infty}(\delta)+\omega_{\infty}(\eta)$.

(iii) The tied spectrum interlaces the periodic spectrum so $\lambda_{2 j-1} \leq \mu_{j} \leq \lambda_{2 j}$ and we can apply (i). In the following result, we will deal with the ambiguity associated with the choice of $\varepsilon_{j}=\mp 1$.

Definition (Jacobian) Regarding Div as a subgroup of $V^{*}$ under the map $J$ of Lemma 7.1(i), we define the real Jacobian of $\mathcal{E}$ to be the abelian group $\mathbf{X}=\operatorname{Div} / \Lambda$. Then $J(\delta)$ has coordinates $J(\delta)=\left(x_{j}(\delta)\right)_{j=-\infty}^{\infty}$ with respect to the sampling sequence. Also let $\mathbf{X}_{0}=\operatorname{Div}_{0} /\left(\Lambda \cap \operatorname{Div}_{0}\right)$.

The inverse spectral problem involves recovering $q$ from the spectral data consisting of the periodic spectrum $\lambda=\left\{\lambda_{j}: j=0,1, \ldots\right\}$ and the family of tied spectra $\left\{\mu_{j}(s): j \in \mathbf{N} ; s \in\right.$ $[0,2 \pi]\}$ of the translated potentials $q(x+s)$ for $s \in[0,2 \pi]$. This gives $\left(\mathbf{p}_{j}-\mathbf{q}_{j}(s)\right) \in \operatorname{Div}$, where $\mathbf{q}_{j}(s)=\left(\mu_{j}(s), \varepsilon \sqrt{\Delta\left(\mu_{j}(s)\right)^{2}-4}\right)$ is a real point on $\mathcal{E}$.

Proposition 7.2 (i) The real periods of $\omega_{\infty}$ vanish, so that $\omega_{\infty}\left(\left(\lambda_{2 j}, 0\right)-\left(\lambda_{2 j-1}, 0\right)\right)=0$ for all $j$. Hence $\omega_{\infty}$ induces a homomorphism $\mathbf{X}_{0} \rightarrow \mathbf{R}$.

(ii) The functions $q(x+s) \mapsto \omega_{\infty}\left(\mathbf{p}_{j}-\mathbf{q}_{j}(s)\right)$ are continuous $\Omega_{N} \rightarrow \mathbf{R}$. The mean value of $(d / d s) \omega_{\infty}\left(\mathbf{p}_{j}-\mathbf{q}_{j}(s)\right)$ with respect to $\nu_{N}^{\beta}$ is zero.

(iii) The map $q \mapsto\left(\omega_{\infty}\left(\mathbf{p}_{j}-\mathbf{q}_{j}(s)\right): j=1,2, \ldots ; s \in[0,2 \pi]\right)$ is one-to-one on $\mathcal{M}_{\lambda}$.

Proof. (i) Observe that $\Delta\left(\lambda_{2 j}\right)=\Delta\left(\lambda_{2 j-1}\right)$, so $\int_{\lambda_{2 j-1}}^{\lambda_{2 j}} \Delta^{\prime}(\lambda) d \lambda / \sqrt{\Delta(\lambda)^{2}-4}=0$. The rest follows from Lemma 7.1(ii). 
(ii) The map $q \mapsto \Delta$ is continuous from the norm topology to the uniform topology on compact planar sets; also $q \mapsto \mu_{j}$ is continuous by Lemma 4.1. Hence $q \mapsto \cosh ^{-1} \Delta\left(\mu_{j}\right)$ is continuous. By (2.2), the measure $\nu_{N}^{\beta}$ is invariant under the translation $q(x) \mapsto q(x+$ $s$ ), and the translation preserves the periodic spectrum. Hence by linearity the mean of $\Delta^{\prime}\left(\mu_{j}(s)\right) \mu_{j}^{\prime}(s) / \sqrt{\Delta\left(\mu_{j}(s)\right)^{2}-4}$ with respect to $\nu_{N}^{\beta}$ equals the mean of

$$
\int_{0}^{2 \pi} \frac{\Delta^{\prime}\left(\mu_{j}(s)\right) \mu_{j}^{\prime}(s) d s}{\sqrt{\Delta\left(\mu_{j}(s)\right)^{2}-4}}
$$

Now as $s$ ranges over $[0,2 \pi], \mu_{j}(s)$ describes $\left[\lambda_{2 j-1}, \lambda_{2 j}\right]$ back and forth an integral number of times, returning to $\mu_{j}(0)$. So by (i), (7.9) equals zero, hence the mean of $(d / d s) \omega_{\infty}\left(\mathbf{p}_{j}-\mathbf{q}_{j}(s)\right)$ equals zero.

(iii) Let $\lambda_{j}^{\prime} \in\left(\lambda_{2 j-1}, \lambda_{2 j}\right)$ satisfy $\Delta^{\prime}\left(\lambda_{j}^{\prime}\right)=0$. Then the graph of $s \mapsto \omega_{\infty}\left(\mathbf{p}_{j}-\mathbf{q}_{j}(s)\right)$ crosses the axis when $\mu_{j}=\lambda_{2 j-1}$ and has height $\cosh ^{-1}\left(\Delta^{\prime}\left(\lambda_{j}^{\prime}\right) / 2\right)$, known as the spike height. In turn, the points $\mu_{j}(s)$ of the tied spectrum are determined by $\cosh ^{-1}\left(\Delta\left(\mu_{j}(s)\right) / 2\right)$.

Given the periodic and tied spectra, we note that by page 329 of [31] for a simple periodic eigenvalue $\lambda_{k}$, there exists a corresponding periodic eigenfunction $f$ such that

$$
-\Delta^{\prime}\left(\lambda_{k}\right) f^{2}(s)=\prod_{j=1}^{\infty} \frac{4\left(\mu_{j}(s)-\lambda_{k}\right)}{j^{2}} .
$$

Finally, one can in principle recover $q$ from

$$
4 f^{4}(s)\left(q(s)-\lambda_{k}\right)=2 f^{2}(s)\left(f^{2}(s)\right)^{\prime \prime}-\left(\left(f^{2}(s)\right)^{\prime}\right)^{2} .
$$

Remark. Whereas typical $q \in\left(\Omega_{N}, \nu_{N}^{\beta}\right)$ are unbounded, one can improve upon Proposition 7.2 (iii) in the case in which $q$ is smooth. Then the differences $\lambda_{2 j}-\lambda_{2 j-1}$ are rapidly decreasing as $j \rightarrow \infty$, and the spike heights are then summable. Trubowitz [31] showed that $q$ may be recovered from the spectral data via

$$
q(s)-\int_{0}^{2 \pi} q(x) \frac{d x}{2 \pi}=-2 \frac{d}{d s} \sum_{j=1}^{\infty} \omega_{\infty}\left(\mathbf{p}_{j}-\mathbf{q}_{j}(s)\right) \quad(s \in[0,2 \pi]) .
$$

Equivalently, $q$ can be recovered from the periodic spectrum, the tied spectrum and so-called norming constants.

In the classical theory of finite genus [16, p.64], the Jacobian map has a nonzero determinant, under certain conditions on the divisors. The following Theorem 7.3 introduces a determinant and gives criterion for the linear statistic associated with sampling at $\left(t_{j}\right)$ to arise from a divisor on $\mathcal{E}$ in the sense of Lemma 7.1 . 
By construction, $\mathbf{X}$ is a infinite-dimensional torus, and has a system of real coordinates. We introduce $\eta_{j}^{0} \in\left[\lambda_{2 j-1}, \lambda_{2 j}\right]$ and then $\eta_{j}\left(s_{j}\right) \in\left[\lambda_{2 j-1}, \lambda_{2 j}\right]$ by the condition

$$
\int_{\eta_{j}\left(s_{j}\right)}^{\lambda_{2 j}} \frac{d x}{\sqrt{\Delta(x)^{2}-4}}=1-s_{j}
$$

where $\eta_{j}(0)=\eta_{j}^{0}$ and $s_{j}$ is a new real variable. Let

$$
L_{0}=\left(\sum_{j=1}^{\infty}\left(\frac{\lambda_{2 j}-\eta_{j}^{0}}{\sqrt{\lambda_{2 j}}+\sqrt{\eta_{j}^{0}}}\right)^{2}\right)^{1 / 2}
$$

We recall from section 6 the biorthogonals $\left(g_{k}\right)_{k \in \mathbf{Z}}$ of the Riesz basis $\left(\operatorname{sinc}\left(s-t_{k}\right)\right)_{k \in \mathbf{Z}}$. For $\sigma=\left(s_{j}\right)_{j=1}^{\infty}$ let $X(\sigma)=J\left(\sum_{j} \delta_{\lambda_{2 j}}-\delta_{\mu_{j}\left(s_{j}\right)}\right)-(1)$, so $X(\sigma)=\left(X_{k}(\sigma)\right)_{k=-\infty}^{\infty}$ where

$$
X_{k}(\sigma)=-1+\sum_{j=1}^{\infty} \int_{\eta_{j}\left(s_{j}\right)}^{\lambda_{2 j}} \frac{g_{k}(\sqrt{x})+g_{k}(-\sqrt{x})}{\sqrt{\Delta(x)^{2}-4}} d x
$$

Theorem 7.3 (i) There exists $C_{\infty}$ such that $\|X(0)\|_{\ell^{\infty}} \leq C_{\infty} \sqrt{\pi} M_{1} L_{0}$, where $\int L_{0} d \nu_{N}^{\beta} \rightarrow 0$ as $N \rightarrow 0$. The Fréchet derivative $X^{\prime}(0)$ defines a bounded linear operator on $\ell^{\infty}$.

(ii) On $\ell^{2}$, the operator $X^{\prime}(0)-I$ is Hilbert-Schmidt and exists $C_{2}$ such that the Carleman determinant satisfies

$$
\left|\operatorname{det}_{2} X^{\prime}(0)-1\right| \leq C_{2} M_{1} L_{0}
$$

(iii) Suppose there exists $\sigma=\left(s_{j}\right)$ in the unit ball of $\ell^{\infty}$ such that $X(\sigma)=0$. Then

$$
\sum_{j=-\infty}^{\infty} g\left(t_{j}\right)=\sum_{j=1}^{\infty} \int_{\eta_{j}\left(s_{j}\right)}^{\lambda_{2 j}} \frac{g(\sqrt{x})+g(-\sqrt{x})}{\sqrt{\Delta(x)^{2}-4}} d x
$$

for all $g$ in the linear span of the $\left(g_{k}\right)_{k \in \mathbf{Z}}$.

Proof. (i) We observe that

$$
L_{0} \leq\left(\sum_{j=1}^{\infty}\left(\frac{\lambda_{2 j}-\lambda_{2 j-1}}{\sqrt{\lambda_{2 j}}+\sqrt{\lambda_{2 j-1}}}\right)^{2}\right)^{1 / 2}
$$

which converges by Proposition 3.1. Also $\lambda_{2 j}-\lambda_{2 j-1} \rightarrow 0$ as $N \rightarrow 0$, so $L_{0} \rightarrow 0$. By the mean value theorem for integrals, there exists $\nu_{j, k} \in\left(\eta_{j}^{0}, \lambda_{2 j}\right)$ such that

$$
X_{k}(0)=\sum_{j=1}^{\infty}\left(g_{k}\left(\sqrt{\nu_{j, k}}\right)+g_{k}\left(-\sqrt{\nu_{j, k}}\right)-g_{k}\left(\sqrt{\lambda_{2 j}}\right)-g_{k}\left(-\sqrt{\lambda_{2 j}}\right)\right) \int_{\eta_{j}^{0}}^{\lambda_{2 j}} \frac{d x}{\sqrt{\Delta(x)^{2}-4}}
$$


where all of the integrals are equal to unity. Then by the mean value theorem, there exist $\omega_{j, k} \in\left(\nu_{j, k}, \lambda_{2 j}\right)$ such that

$$
X_{k}(0)=\sum_{j=0}^{\infty}\left(g_{k}^{\prime}\left(\sqrt{\omega_{j, k}}\right)-g_{k}^{\prime}\left(-\sqrt{\omega_{j, k}}\right)\right)\left(\sqrt{\omega_{j, k}}-\sqrt{\lambda_{2 j}}\right)
$$

so by the Cauchy-Schwarz inequality

$$
\left|X_{k}(0)\right| \leq\left(\sum_{j=1}^{\infty}\left(g_{k}^{\prime}\left(\sqrt{\omega_{j, k}}\right)^{2}+g_{k}^{\prime}\left(-\sqrt{\omega_{j, k}}\right)^{2}\right)^{1 / 2}\left(\sum_{j=1}^{\infty}\left(\sqrt{\lambda_{2 j}}-\sqrt{\omega_{j, k}}\right)^{2}\right)^{1 / 2}\right.
$$

and since $\left( \pm \sqrt{\omega_{j, k}}\right)$ gives a sampling sequence for $R P W(\pi)$ we can choose $C_{\infty}$ independent of $k$ such that

$$
\left|X_{k}(0)\right| \leq C_{\infty}\left\|g_{k}^{\prime}\right\|_{L^{2}}\left(\sum_{j=1}^{\infty}\left(\frac{\lambda_{2 j}-\eta_{j}^{0}}{\sqrt{\lambda_{2 j}}+\sqrt{\eta_{j}^{0}}}\right)^{2}\right)^{1 / 2}
$$

hence $X(0)=\left(X_{k}(0)\right)$ is bounded and $\|X(0)\|_{\ell^{\infty}} \leq C_{\infty} \sqrt{\pi} M_{1} L_{0}$.

The function $X: \operatorname{Ball}\left(\ell^{\infty}\right) \rightarrow \ell^{\infty}$ is Fréchet differentiable near to $s=0$, and the derivative is expressed as an infinite matrix with respect to the usual weak* basis

$$
X^{\prime}(s)=\left[\frac{\partial X_{k}}{\partial s_{j}}\right]_{j, k}=\left[g _ { k } \left(\sqrt{\eta_{j}\left(s_{j}\right)}+g_{k}\left(-\sqrt{\eta_{j}\left(s_{j}\right)}\right]\right.\right.
$$

so in particular

$$
X^{\prime}(0)-\left[\delta_{j, \pm k}\right]=\left[g_{k}\left(\sqrt{\eta_{j}^{0}}\right)+g_{k}\left(-\sqrt{\eta_{j}^{0}}\right)-g_{k}\left(\sqrt{\lambda_{2 j}}\right)-g_{k}\left(-\sqrt{\lambda_{2 j}}\right)\right]
$$

The rows of this matrix are absolutely summable with uniformly bounded sums, so as in (7.20), $X^{\prime}(0)$ defines a bounded linear operator on $\ell^{\infty}$.

(ii) Furthermore, $\left(g_{k}\right)$ is itself a Riesz basis, and hence for any pair of real sequences $\left(u_{j}\right)$ and $\left(v_{j}\right)$ such that $\left(u_{j}-v_{j}\right) \in \ell^{2}$, we have

$$
\sum_{j, k}\left|g_{k}\left(u_{j}\right)-g_{k}\left(v_{j}\right)\right|^{2} \leq M_{1}^{2} \sum_{j}\left(u_{j}-v_{j}\right)^{2}
$$

as in (3.21), where the final series converges. In particular, we can take $u_{j}=\sqrt{\eta_{j}^{0}}$ and $v_{j}=\sqrt{\lambda_{2 j}}$, so that $g_{k}\left(v_{j}\right)=\delta_{k, j}$. Hence $X^{\prime}(0)-I$ is a Hilbert-Schmidt operator, with norm bounded by a constant multiple of $M_{1} L_{0}$. Hence $X^{\prime}(0)$ has a Carleman determinant, and det $_{2}$ is a Lipschitz continuous function on bounded subsets of $I+H S$. (We have not quite proved that $X^{\prime}(0)$ has a Hill's determinant as in [20, p. 29], since (7.20) involves sums of squares.) 
(iii) We observe that the biorthogonal system satisfies

$$
\sum_{j=-\infty}^{\infty} g_{k}\left(t_{j}\right)=\sum_{j=-\infty}^{\infty}\left\langle g_{k}(x), \operatorname{sinc}\left(x-t_{j}\right)\right\rangle_{L^{2}}=1
$$

since only one term in the sum is nonzero. Hence the condition $X_{k}(s)=0$ gives the identity (7.17) for $g=g_{k}$, and the general case follows by linearity.

Corollary 7.4 (i) Suppose $C_{\infty} \sqrt{\pi} M_{1} L_{0}<1 / 3$. Then $X^{\prime}(0)$ defines an invertible linear operator on $\ell^{\infty}$.

(ii) Suppose further that

$$
\left\|X(s)-X(0)-X^{\prime}(0) s\right\|_{\ell^{\infty}} \leq(1 / 6)\|s\|_{\ell^{\infty}} \quad\left(s \in \operatorname{Ball}_{1}\left(\ell^{\infty}\right)\right) .
$$

Then the sequence $\left(\sigma_{n}\right)_{n=1}^{\infty}$ produced by Newton's modified algorithm $\sigma_{0}=0$ and

$$
\sigma_{n+1}=\sigma_{n}-X^{\prime}(0)^{-1} X\left(\sigma_{n}\right), \quad(n=0,1, \ldots)
$$

converges to $\sigma$ such that $X(\sigma)=0$, so Theorem 7.3(iii) holds.

Proof. (i) When $C_{\infty} \sqrt{\pi} M_{1} L_{0}<1$, the operator $X^{\prime}(0)$ on $\ell^{\infty}$ satisfies $\left\|X^{\prime}(0)-I\right\|<1$ by Theorem 7.2(i), and hence $X^{\prime}(0)$ is invertible with $\left\|X^{\prime}(0)^{-1}\right\| \leq\left(1-C_{\infty} \sqrt{\pi} M_{1} L_{0}\right)^{-1}$.

(ii) This follows from (iv) by Corollary 2 of [1].

Acknowledgements The first-named author carried out part of this work during a visit to the University of New South Wales. He also thanks H.P. McKean for a motivating discussion. The work of the second-named author was partially supported by an EPSRC research studentship. The work of the third-named author was partially supported by an LMS Scheme 2 grant. The authors thank Nadia Mazza for language assistance.

\section{References}

[1] R.G. Bartle, Newton's method in Banach spaces, Proc. Amer. Math. Soc. 6 (1955), $827-831$.

[2] G. Blower, Almost sure weak convergence for the generalized orthogonal ensemble, J. Statist. Phys. 105 (2001), 309-335.

[3] G. Blower, A logarithmic Sobolev inequality for the invariant measure of the periodic Korteweg-de Vries equation, Stochastics 84 (2012), 533-542.

[4] G. Blower, C. Brett and I. Doust, Logarithmic Sobolev inequalities and spectral concentration for the periodic Schrödinger equation, Stochastics 86 (2014), 870-881.

[5] S.G. Bobkov, I. Gentil and M. Ledoux, Hypercontractivity of Hamilton-Jacobi equations, J. Math. Pures Appl. (9) 80 (2001), 669-696. 
[6] J. Bourgain, Fourier transform restriction phenomena for certain lattice subsets and applications to nonlinear evolution equations II: The KdV equation, Geom. Funct. Anal. 3 (1993), 209-262.

[7] J. Bourgain, Periodic nonlinear Schrödinger equation and invariant measures, Comm. Math. Phys. 166 (1994), 1-26.

[8] A. Erdelyi, Über die freien Schwingungen in Kondensatorkreisen mit periodisch verändlicher Kapaziteier, Ann. der Physik 5 folge band 19 (1934), 585-622.

[9] C. S. Gardner, J. M. Greene, M. D. Kruskal, and R. M. Miura, Korteweg-de Vries equation and generalization VI. Methods for exact solution. Comm. Pure Appl. Math. 27 (1974), 97-133.

[10] J. Garnett and E. Trubowitz, Gaps and bands of one-dimensional periodic Schrödinger operators, Comment. Math. Helvetici 59 (1984), 258-312.

[11] J.R. Higgins, Five short stories about the cardinal series, Bull. Amer. Math. Soc. NS 12 (1985), 45-89.

[12] K. Johansson, On random matrices from the compact classical groups, Ann. of Math. (2) 145 (1997), 519-545.

[13] T. Kappeler and B. Mityagin, Gap estimates of the spectrum of Hill's equation and action variables for KdV, Trans. Amer. Math. Soc. 351 (1999), 619-646.

[14] N.M. Katz and P. Sarnak, Random matrices, Frobenius eigenvalues and monodromy, (American Mathematical Society, Providence, R.I. 1999).

[15] P. Koosis, The logarithmic integral I, (Cambridge University Press, 1988).

[16] S. Lang, Introduction to algebraic and abelian functions, Second Edition, (Springer-Verlag, New York, 1982).

[17] P. D. Lax, Integrals of nonlinear equations of evolution and solitary waves, Comm. Pure Appl. Math. 21 (1968), 467-490.

[18] J.L. Lebowitz, Ph. Mouniax and W.-M. Wang, Approach to equilibrium for the stochastic NLS, Comm. Math. Phys. 321 (2013), 69-84.

[19] A. Lytova and L Pastur, Central Limit Theorem for linear eigenvalue statistics of random matrices with independent entries, Ann. Probab. 37 (2009), 1778-1840.

[20] W. Magnus and S. Winkler, Hill's Equation, second edition (Dover Publications, New York, 1979).

[21] H.P. McKean and P. van Moerbeke, The spectrum of Hill's equation, Invent. Math. 30 (1975), 217-274.

[22] H.P. McKean and E. Trubowitz, Hill's operator and hyperelliptic function theory in the presence of infinitely many branch points, Comm. Pure Appl. Math. 29 (1976), 143-226.

[23] J. Moser, Various aspects of integrable Hamiltonian systems, 233-289, Dynamical Systems, (Birkhauser, Boston, 1980). 
[24] N.K. Nikolski, Operators, Functions and Systems: An Easy Reading Vol. 2: Model Operators and Systems, (American Mathematical Society, Providence, R.I., 2002).

[25] J. Ortega-Cerdà and K. Seip, Fourier frames, Ann. of Math. (2) 155 (2002), 789-806.

[26] J.R. Partington, Interpolation, identification and sampling (Oxford University Press, 1997).

[27] H.L. Pedersen, Entire functions having small logarithmic sums over certain discrete subsets, Ark. Mat. 36 (1998), 119-130.

[28] B. Simon, Trace ideals and their applications, (Cambridge University Press, 1979).

[29] A Soshnikov, The Central Limit Theorem for local linear statistics in classical compact groups and related combinatorial identities, Ann. Probab. 28 (2000), 1353-1370. [30] C.A. Tracy and H. Widom, Correlation functions, cluster functions and spacing distributions for random matrices, J. Statist. Phys. 92 (1998), 809-835.

[31] E. Trubowitz, The inverse problem for periodic potentials, Comm. Pure Appl. Math. 30 (1977), 321-337.

[32] C. Villani, Topics in Optimal Transportation (American Mathematical Society, Providence R.I., 2003).

[33] C. Villani, Optimal transport, old and new, (Springer-Verlag, 2009). 